\title{
Verification of bubble tracking method and DNS examinations of single- and two-phase turbulent channel flows
}

Jun Fang, NCSU

Igor A. Bolotnov, NCSU

Jiacai Lu, University of Notre Dame Gretar Tryggvason, University of Notre Dame

March 30, 2017

Approved for Public Release
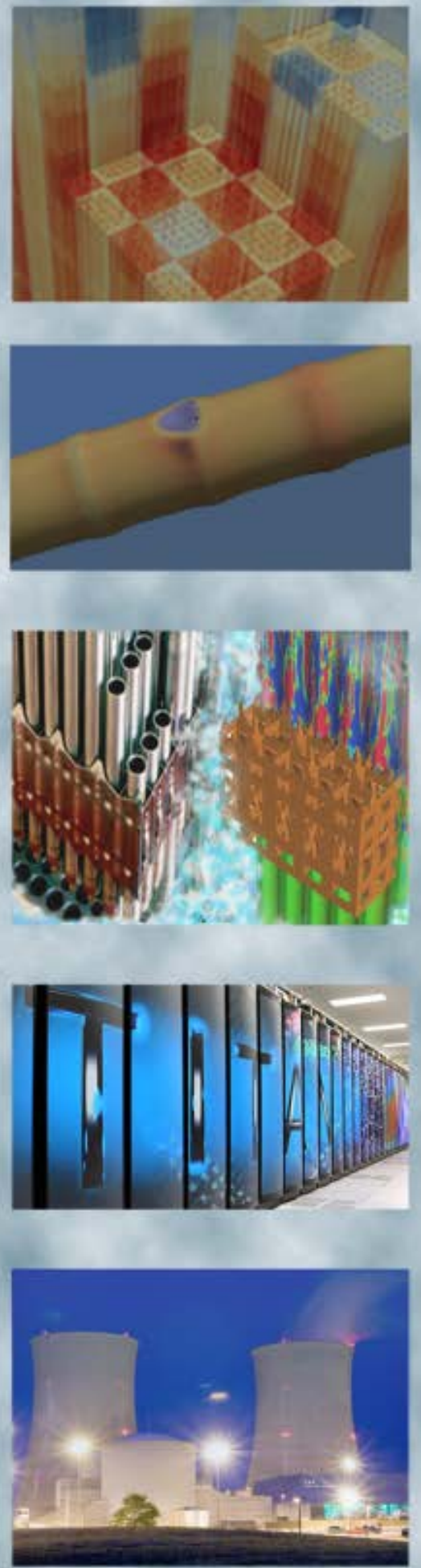
Please complete sections appropriate for this record.

REVISION LOG

\begin{tabular}{|c|c|c|l|}
\hline Revision & Date & Affected Pages & \multicolumn{1}{c|}{ Revision Description } \\
\hline 0 & $03 / 30 / 2017$ & All & Initial Release \\
\hline & & & \\
\hline & & & \\
\hline & & & \\
\hline
\end{tabular}

\section{Document pages that are:}

Export Controlled ___None

IP/Proprietary/NDA Controlled____None

Sensitive Controlled___ None

Unlimited

All

\section{Requested Distribution:}

To:

Copy:

This report was prepared as an account of work sponsored by an agency of the United States Government. Neither the United States Government nor any agency thereof, nor any of their employees, makes any warranty, express or implied, or assumes any legal liability or responsibility for the accuracy, completeness, or usefulness of any information, apparatus, product, or process disclosed, or represents that its use would not infringe privately owned rights. Reference herein to any specific commercial product, process, or service by trade name, trademark, manufacturer, or otherwise, does not necessarily constitute or imply its endorsement, recommendation, or favoring by the United States Government or any agency thereof. The views and opinions of authors expressed herein do not necessarily state or reflect those of the United States Government or any agency thereof. 


\title{
Verification of bubble tracking method and DNS examinations of single- and two-phase turbulent channel flows
}

\section{L3:THM.CLS.P14.03 milestone report}

\author{
Jun Fang ${ }^{1}$ \\ Igor A. Bolotnov ${ }^{1}$ \\ Jiacai $\mathrm{Lu}^{2}$ \\ Gretar Tryggvason ${ }^{2}$
}

${ }^{1}$ North Carolina State University
${ }^{2}$ University of Notre Dame

March 2017 


\section{Contents}

LIST OF FIGURES .........................................................................

LIST OF TABLES ................................................................... 4

1. ReleVAnCE to CASL AND OBJeCtIVES ......................................... 5

2. DESCRIPTION OF NUMERICAL CODES ........................................... 5

3. Sensitivity Study of Data Collection TeChNiques in BubBle

TRACKING SIMULATIONS ...........................................................6

4. High-Fidelity Simulations OF TURBULENT FloWs In PWR SUBCHANNEL GEOMETRY WITH SPACER GRID AND MixING VANES. ................................. 8

4.1 SIMULATION SETUP ............................................................... 9

4.2 RESULTS AND DISCUSSION .............................................. 11

5. USING DNS to EXAMINE TURBULENT BUbBLy FLOW IN VERTICAL

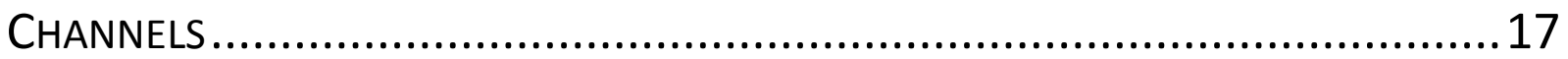

5.1 DNS DATABASES FOR BUBBLY FLOWS....................................... 17

5.2 EFFECT OF SURFACTANTS ON DEFORMABLE BUBBLES.........................20

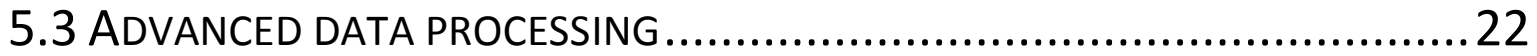

5.4 PUBLICATIONS IN PREPARATION ................................................. 27

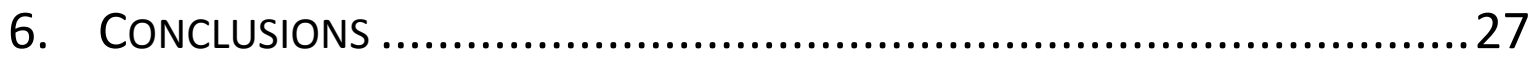

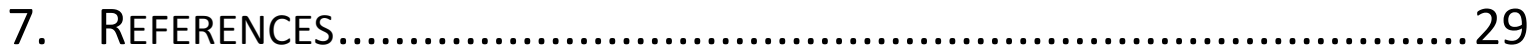




\section{List of Figures}

Fig 1. The steady state bubble position in the domain. ................................................................. 6

Fig 2. The extracted bubble relative velocity from liquid shell with different thicknesses................... 7

Fig 3. The extracted bubble relative velocity from liquid shell with the same thickness at various locations

Fig 4. The geometric models of three investigated simulation cases (front walls are hided to show the inner structures); dashed lines marked illustrate the locations of measurement planes (MP)............ 9

Fig 5. The virtual probe plane to record instantaneous turbulence quantities. ................................ 11

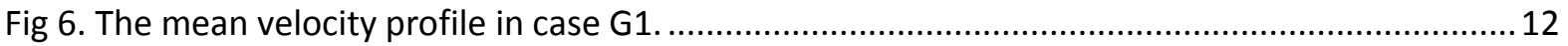

Fig 7. The non-dimensional mean flow velocity profile in case G1............................................... 12

Fig 8 . The turbulence vortices induced by the spacer grid and mixing vanes.................................... 13

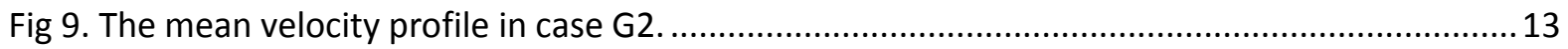

Fig 10. The non-dimensional mean flow velocity profile in case G2 ............................................ 14

Fig 11. The instantaneous turbulence velocity field of the last time step at all measurement planes in domain $\mathrm{G} 3$

Fig 12. The mean flow velocity profiles at different downstream locations as well as the free subchannel (G1).

Fig 13. The turbulent kinetic energy profiles at different downstream locations as well as the free

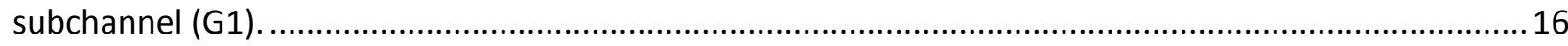

Fig 14. The highlighted location of observed TKE peak with respect to the ................................... 16

Fig 15. Showing the bubbles (left) and the bubbles and iso-surfaces of lambda-2 $=-1.5$ at time 500 (right).

Fig 16. The average wall shear (top) and the flow rate (bottom) versus time.................................. 18

Fig 17. Several quantities averaged over planes parallel to the walls, versus the wall normal coordinate.

Fig 18. Two frames from simulations of deformable bubbles. In the left frame the surface tension is constant, but in the right frame it is a function of the concentration of insoluble surfactant. .................21

Fig 19. The average wall shear (top) and the flow rate (bottom) versus time.................................... 21

Fig 20. The energy of the modes for POD. The top frame shows the energy ratio (the energy in each mode divided by the total energy) and the bottom frame shows the accumulated energy versus the mode number, arranged in order of decreasing energy.

Fig 21. Original data (left). Reconstruction using 120 POD modes (right). ........................................23

Fig 22. Reconstruction using the first POD mode only (left). Reconstruction using twenty POD modes

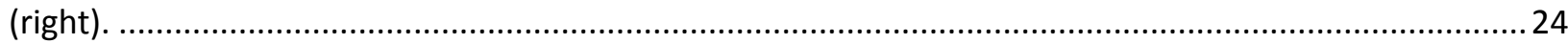

Fig 23. Distribution of Eigenvalues $\mu_{1}$ of all dynamic modes, as computed by the standard DMD. .... 25

Fig 24. Dependence of the DMD amplitudes on the spectra (Left) and the real part (Right) of the corresponding DMD eigenvalues, as computed by the standard DMD. .................................................2 25

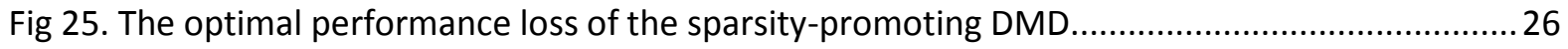

Fig 26. Eigenvalues (red circles) from the subset of $\mathrm{N}$ modes selected by the sparsity-promoting DMD

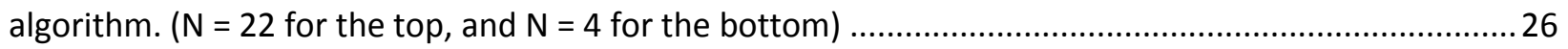


Fig 27. Dependence the amplitudes on the frequency for the subset of $\mathrm{N}$ modes selected by the sparsity-promoting DMD algorithm (red circles) with $\mathrm{N}=4$ for the left and $\mathrm{N}=22$ for the right.

\section{List of Tables}

Table 1: The marked local liquid region with different thickness. ............................................ 7

Table 2: The marked local liquid region with the same thickness and different locations................... 7

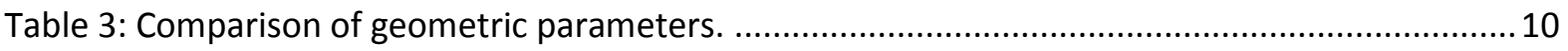

Table 4: The locations of measurement probe planes in domain G3 ............................................. 10

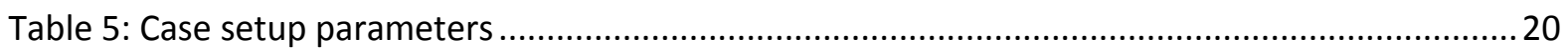




\section{Relevance to CASL and Objectives}

Description: Direct numerical simulation (DNS) has been regarded as a reliable data source for the development and validation of turbulence models along with experiments. The realization of DNS usually involves a very fine mesh that should be able to resolve all relevant turbulence scales down to Kolmogorov scale [1]. As the most computationally expensive approach compared to other CFD techniques, DNS applications used to be limited to flow studies at very low Reynolds numbers. Thanks to the tremendous growth of computing power over the past decades, the simulation capability of DNS has now started overlapping with some of the most challenging engineering problems. One of those examples in nuclear engineering is the turbulent coolant flow inside reactor cores. Coupled with interface tracking methods (ITM), the simulation capability of DNS can be extended to more complicated two-phase flow regimes.

Departure from nucleate boiling (DNB) is the limiting critical heat flux phenomena for the majority of accidents that are postulated to occur in pressurized water reactors (PWR) [2]. As one of the major modeling and simulation (M\&S) challenges pursued by CASL, the prediction capability is being developed for the onset of DNB utilizing multiphase-CFD (M-CFD) approach. DNS (coupled with ITM) can be employed to provide closure law information for the multiphase flow modeling at CFD scale. In the presented work, research groups at NCSU and UND will focus on applying different ITM to different geometries. Higher void fraction flow analysis at reactor prototypical conditions will be performed, and novel analysis methods will be developed, implemented and verified for the challenging flow conditions.

\section{Description of Numerical Codes}

This is a joint milestone which utilizes the Front Tracking code (FTC3D) at UND and Level Set based code (PHASTA) at NCSU. The DNS solver being used at NCSU is called PHASTA [3], a computational fluid dynamics package designed for both compressible and incompressible turbulent flows. It is a finite element based code that can support both structured and unstructured meshes. Due to the compatibility with unstructured mesh, PHASTA can simulate the turbulence problems in complicated geometries, like the spacer grid and mixing vanes in current study. Since DNS requires very fine meshes, the flow solver should be able to handle and perform the computations with huge amounts of mesh cells. As an indispensable requirement for DNS solver, the excellent scaling performance of PHASTA has already been demonstrated in massively parallel simulations (up to 786,432 cores) [4]. PHASTA can be also utilized in two-phase flow simulations by incorporating the level set method to track the gas-liquid interface [5]. For the details of code FTC3D please refer to previous publication [6]. 


\section{Sensitivity Study of Data Collection Techniques in Bubble Tracking Simulations}

To make the best use of large-scale high-fidelity interface tracking simulations, the bubble tracking methodology has been developed [7]. It offers the simulation capability to track individual bubbles and extract the important bubble parameters of interest. Some examples of the bubble parameters include the bubble deformation level, local liquid velocity and shear rate a bubble experiencing. These parameters are difficult to collect from experimental measurements especially when turbulence and a huge number of bubbles are involved. In the previous CASL milestone report [8], we have provided detailed introductions upon the development and application of bubble tracking method. In the framework of bubble tracking method, the user is given the flexibility to specify the colored local liquid regions, such as the thickness and/or the location of local liquid shell. In this section, we will evaluate how the extracted bubble tracking data would depend on the size and location of local liquid regions. The controlled single bubble scenario is simulated with various configurations of the local liquid region tracked. The bubble relative velocity and liquid shear rate are to be collected and compared with the prescribed flow conditions. The bubble sees a uniform inflow at a velocity of $0.05 \mathrm{~m} / \mathrm{s}$; the steady state bubble position is shown in Fig 10.

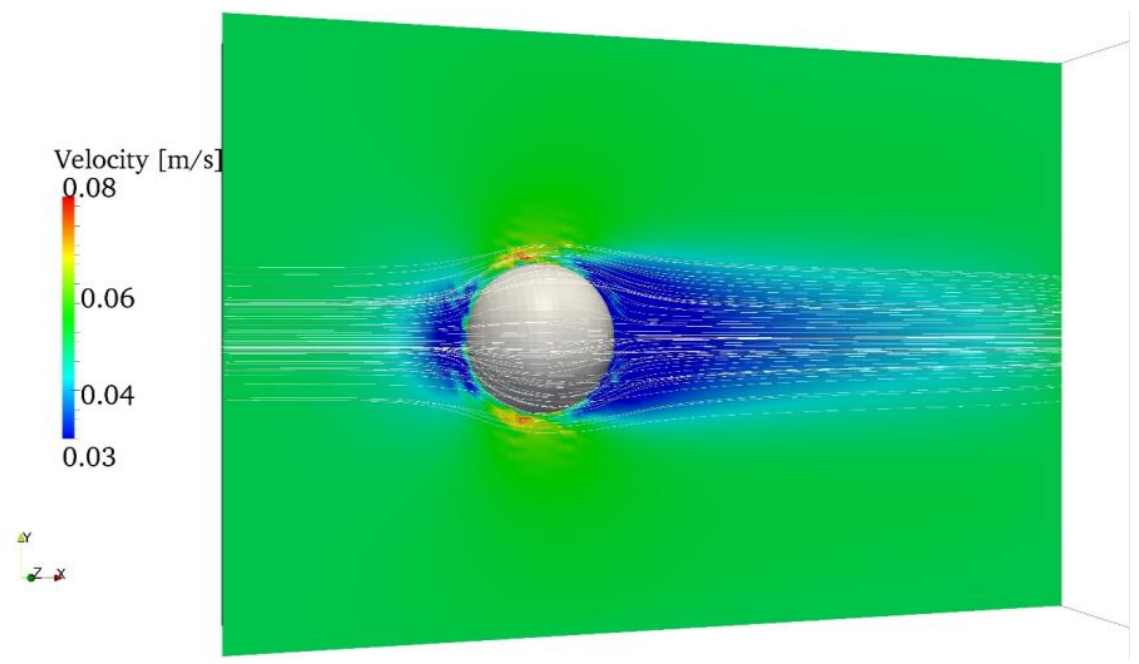

Fig 1 . The steady state bubble position in the domain.

There are two set of test cases to carry out: the first set of cases has the different liquid shell thickness while the inner liquid front is fixed at $1 \varepsilon$ in level set distance field, where the $1 \varepsilon$ length corresponds to half interface thickness $\left(6.0 \times 10^{-5} \mathrm{~m}\right)$. The second set of test cases has the same thickness of colored liquid region but the relative location is different with respect to the bubble interface. For both test sets, the colored liquid regions under steady state are shown in Table 1 and Table 2.

When the bubble is fully controlled under the steady state, the average bubble velocity is very close to zero. Extracted local liquid velocity is also the bubble relative velocity which has the expected value of $0.05 \mathrm{~m} / \mathrm{s}$ in the considered scenario. As shown in Fig 2 and Fig 3, the extracted relative velocities from all 
test cases are close to the expected magnitude and present an asymptotic trend as tracked liquid region becomes thicker and covers more volume outside the bubble interface. In the thickness test set (THI01 to THI05), the largest relative error observed is $13.0 \%$ (from case THI01) while the smallest error is $4.8 \%$ (from THI05). Note that since the bubble relative velocity is not zero, there is a hydrodynamic boundary layer developed outside the bubble interface. The inclusion of this boundary layer region leads to an under-predicted bubble relative velocity.

Table 1: The marked local liquid region with different thickness.

\begin{tabular}{|c|c|c|c|c|c|}
\hline Case name & THI01 & THIO2 & THIO3 & THIO4 & THI05 \\
\hline $\begin{array}{l}\text { Liquid shell } \\
\text { range }\end{array}$ & $1 \varepsilon-2 \varepsilon$ & $1 \varepsilon-3 \varepsilon$ & $1 \varepsilon-4 \varepsilon$ & $1 \varepsilon-5 \varepsilon$ & $1 \varepsilon-6 \varepsilon$ \\
\hline $\begin{array}{l}\text { The marked } \\
\text { liquid } \\
\text { region }\end{array}$ & & & & & \\
\hline
\end{tabular}

Table 2: The marked local liquid region with the same thickness and different locations.

\begin{tabular}{|l|c|c|c|c|}
\hline Case name & LOCO1 & LOC02 & LOCO3 & LOCO4 \\
\hline $\begin{array}{l}\text { Liquid shell } \\
\text { range }\end{array}$ & $1 \varepsilon-3 \varepsilon$ & $2 \varepsilon-4 \varepsilon$ & $3 \varepsilon-5 \varepsilon$ & $4 \varepsilon-6 \varepsilon$ \\
\hline $\begin{array}{l}\text { The colored } \\
\text { liquid } \\
\text { region }\end{array}$ & & & & \\
\hline
\end{tabular}

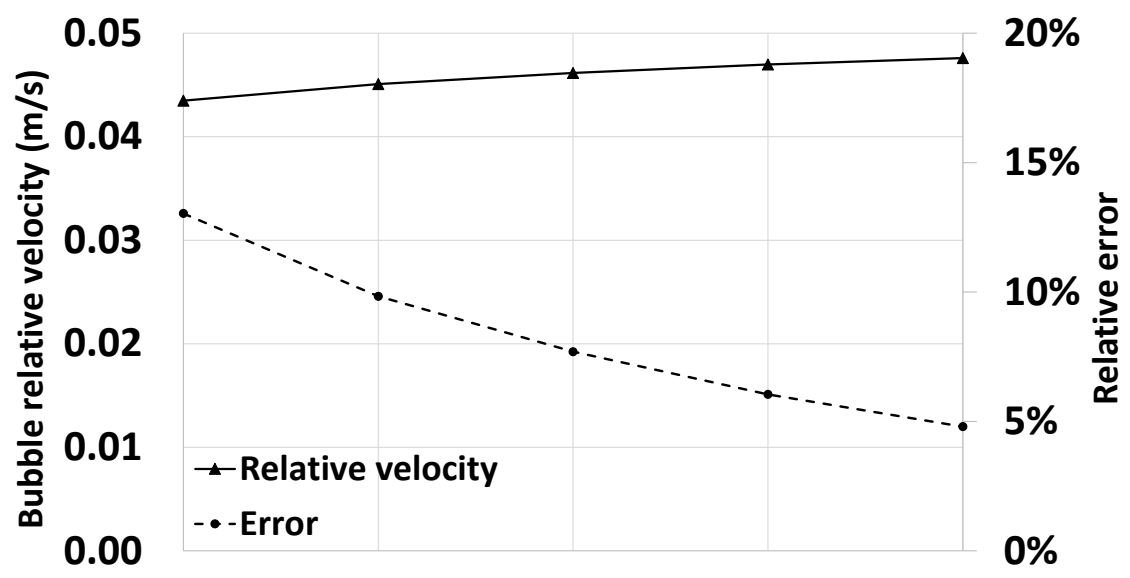
2.0
3.0
4.0
5.0
6.0

Outer front level set value of liquid shell (half interface thickness)

Fig 2. The extracted bubble relative velocity from liquid shell with different thicknesses. 
In the second test set (LOCO1 to LOCO4), the relative error is generally smaller (as shown in Fig 3). This is because that the tracked local liquid region avoids the velocity boundary layer right outside the interface except the case LOC01. In other words, the low velocity region influenced by the presence of bubble is excluded in most of those cases. Therefore, to obtained reliable local liquid information, the colored local liquid region should exclude the velocity boundary layer outside the bubble. In the meantime, the tracked liquid region should be also close enough to the bubble interface in order to truly represent the local flow condition. Based on all test cases, a recommended liquid region range is from $3 \varepsilon$ to $5 \varepsilon$ (i.e. LOCO3) which provides a bubble relative velocity with an error less than $5 \%$.

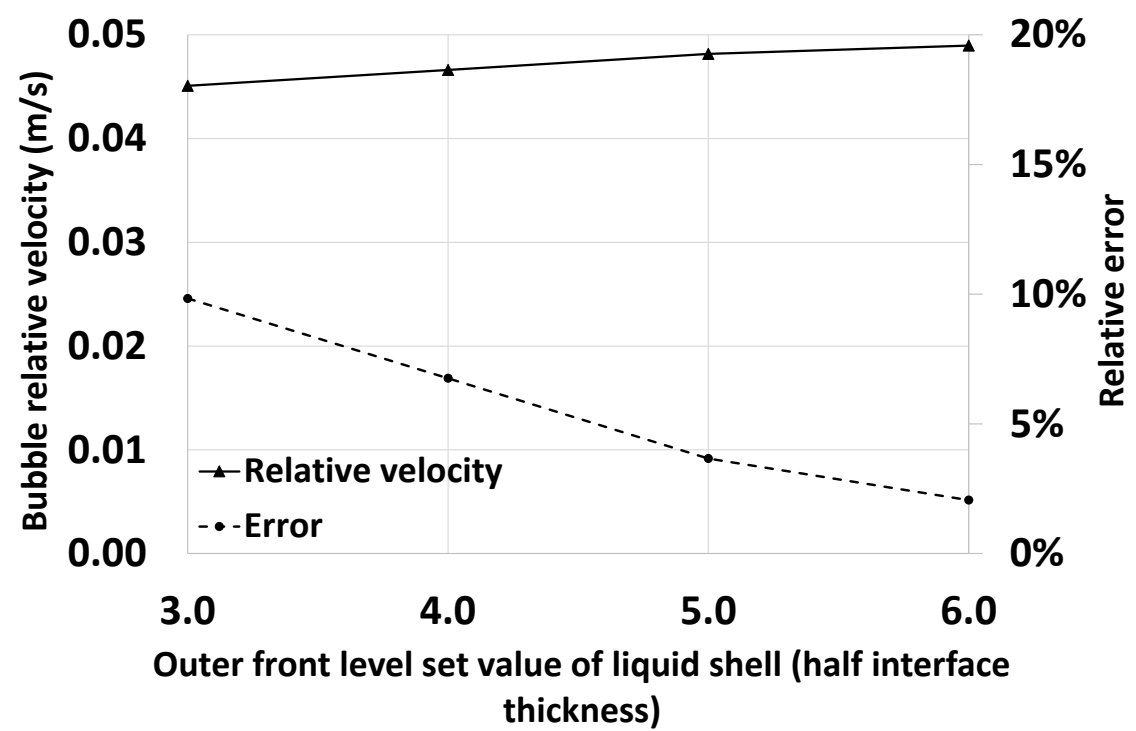

Fig 3. The extracted bubble relative velocity from liquid shell with the same thickness at various locations.

\section{High-fidelity Simulations of Turbulent Flows in PWR Subchannel Geometry with Spacer Grid and Mixing Vanes.}

In Light Water Reactor (LWR) cores, the nuclear fuel rods are arranged together as rod bundles in a triangular or square pattern. The fuel rod bundles are supported by the spacer grids. The mixing vanes are also installed to promote the turbulence intensity in coolant flow. An understanding of spacer grid and mixing vanes effect is important because it has a direct impact on the heat removal efficiency from the fuel rod surface. In the presented work, the DNS of turbulent flows are performed in three distinct types of geometries: (a) a regular PWR subchannel; (b) a special PWR subchannel with reduced size spacer grid and mixing vanes (SGMV); (c) a special PWR subchannel with reduced size SGMV, but with a longer domain size (Fig 4). The springs and dimples of the spacer grid are not represented for the simplicity purpose in present investigation. The mean flow velocity and turbulent kinetic energy profiles are averaged from the instantaneous DNS data in different types of geometries as well as various downstream locations with respect to the SGMV region. The results will help better assess the influence of spacer grid and mixing vanes on core coolant flows, and serve as a reference for the two-phase turbulence results in the same geometries. 


\subsection{Simulation setup}

The PWR subchannel geometry is selected as the computational domain, of which the design is crucial for reactor thermal-hydraulics analyses. There are 3 different types of the subchannel geometry considered in this study: the first type (G1) is a regular subchannel with no internal structures; the second one (G2) is a subchannel geometries with reduced size PWR spacer grid and mixing vanes; the third one (G3) has the same internal structures as $G 2$ but with an extended channel height. In domain $\mathrm{G} 3$, the height ratio between spacer grid and the entire subchannel is kept the same as that one would expect in a realistic PWR subcahnnel geometry. The mean flow velocity is $0.75 \mathrm{~m} / \mathrm{s}$ in all three cases, of which the corresponding hydraulic Reynolds number $\left(R e_{h}\right)$ is about 80,000 . The geometric ratio ensures the turbulence eddies can have the same relative residence time at the spacer grid region in each flow through period as in PWR core operating conditions if one considers the reduced flow rate being modeled. More geometric details are listed in Table 3. Although the investigated Reynolds number is only roughly a sixth of that under the realistic PWR flow condition [5], it is still much higher than what one can find in the literature for DNS studies on reactor coolant flow [9].

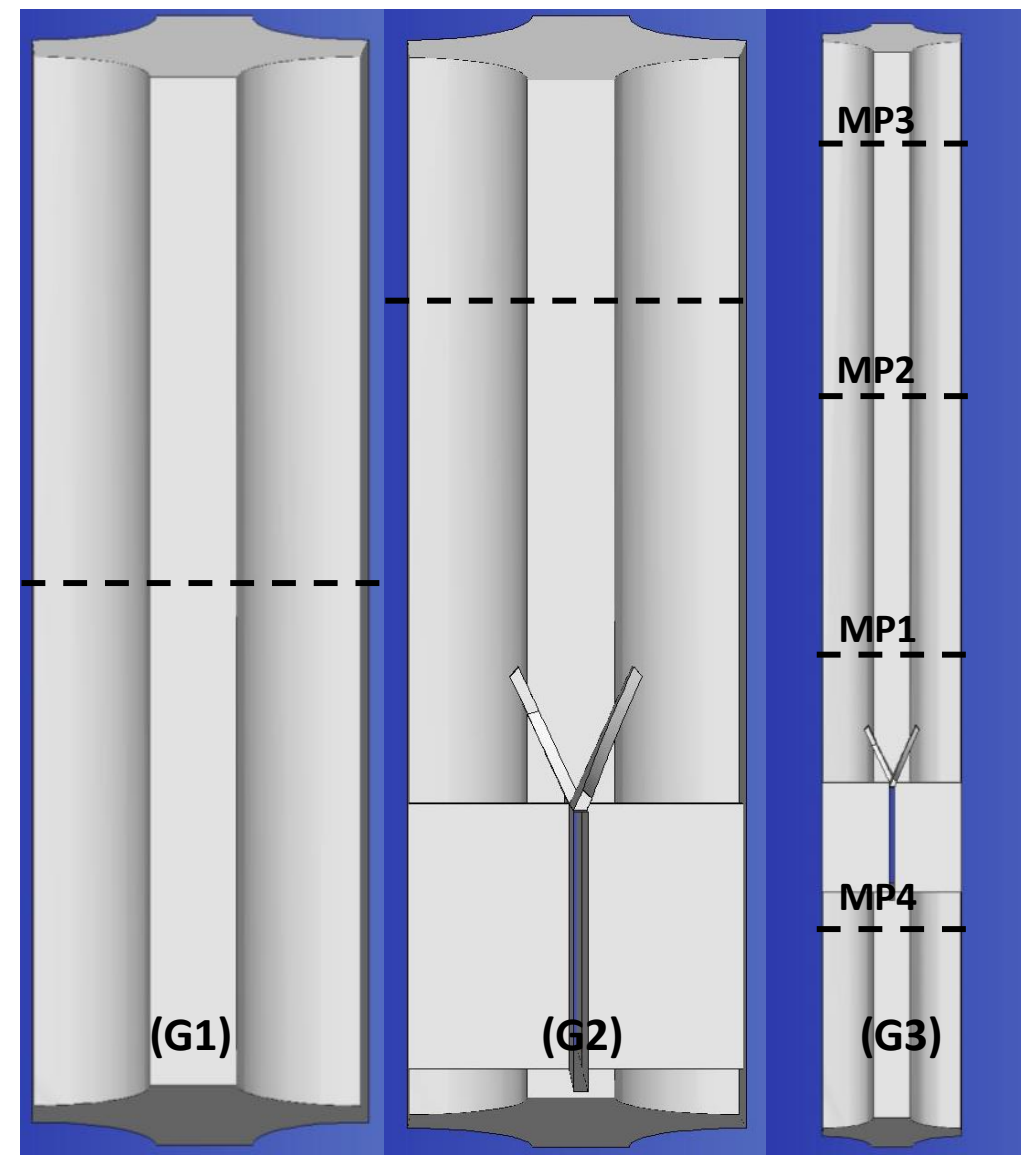

Fig 4. The geometric models of three investigated simulation cases (front walls are hided to show the inner structures); dashed lines marked illustrate the locations of measurement planes (MP). 
In DNS, the grid spacing must be sufficiently fine to capture the small scales of interest. To meet the prerequisite, the mesh cell sizes should be comparable to the Kolmogorov length scale. For example, for the first layer of mesh cells upon the wall, the designed cell size is $3.25 \mu \mathrm{m}$ while at the same location $a$ posteriori Kolmogorov length scale is $2.85 \mu \mathrm{m}$. The Kolmogorov length scale in the entire domain ranges from $2.85 \mu \mathrm{m}$ to $11.7 \mu \mathrm{m}$. The first plane of grid points off the walls has an equivalent distance of about $\Delta y^{+}=1.0$. More discussions regarding the DNS resolution requirements for turbulent flows can be found in [10].

Table 3: Comparison of geometric parameters.

\begin{tabular}{|l|r|r|r|}
\hline Cases & G1: & G2: & G3: \\
\hline Rod radius $(m)$ & \multicolumn{3}{|c|}{$4.57 \times 10^{-3}$} \\
\hline Aspect Ratio $(P / D)$ & \multicolumn{3}{|c|}{1.38} \\
\hline Domain height $(m)$ & 0.0405 & 0.0405 & 0.1 \\
\hline Spacer grid height $(m)$ & $\mathrm{n} / \mathrm{a}$ & 0.01 & 0.005 \\
\hline Mixing vane height $(m)$ & $\mathrm{n} / \mathrm{a}$ & 0.01 & 0.005 \\
\hline
\end{tabular}

The periodic boundary condition is applied to domain inlet/outlet as well as the transverse faces. The non-slip wall condition is applied to both fuel rod surface and the surfaces of spacer grid and mixing vanes. In case G1, a solid obstacle is introduced in the domain to help generate the large velocity fluctuations. Once enough velocity fluctuations are observed, the obstacle is removed, and the simulation carries on till a sustainable turbulence field is achieved. The solid obstacle is not needed in geometries G2 and G3 because the existence of mixing vanes can efficiently create the necessary velocity fluctuations. With enough pressure gradient, the coolant flow through $\mathrm{G} 2$ and $\mathrm{G} 3$ can achieve desired turbulence profiles.

Table 4: The locations of measurement probe planes in domain G3

\begin{tabular}{|l|r|r|}
\hline $\begin{array}{l}\text { Measurement } \\
\text { plane }\end{array}$ & $\begin{array}{r}\text { Distance from the } \\
\text { periodic inlet }(m)\end{array}$ & $\begin{array}{r}\text { Distance from the top of } \\
\text { mixing vanes }(m)\end{array}$ \\
\hline MP1 & 0.05 & 0.01283 \\
\hline MP2 & 0.07 & 0.03283 \\
\hline MP3 & 0.09 & 0.05283 \\
\hline MP4* & 0.01 & 0.07283 \\
\hline
\end{tabular}

To analyze the turbulent flow, a plane of virtual probes is placed in the domain normal to the mean flow direction as shown in Fig 4. By averaging the turbulence quantity in a designated time window, one

\footnotetext{
${ }^{1}$ The aspect ratio is the ratio of distance between two fuel rod centers over the rod diameter.
} 
can obtain the corresponding mean turbulence quantity [11]. Only one probe plane is placed in the domains $\mathrm{G} 1$ and $\mathrm{G} 2$, while four measurement planes are placed in the domain $\mathrm{G} 3$ to assess the impact of SGMV at different downstream locations. The locations of measurement planes are listed in Table 4.

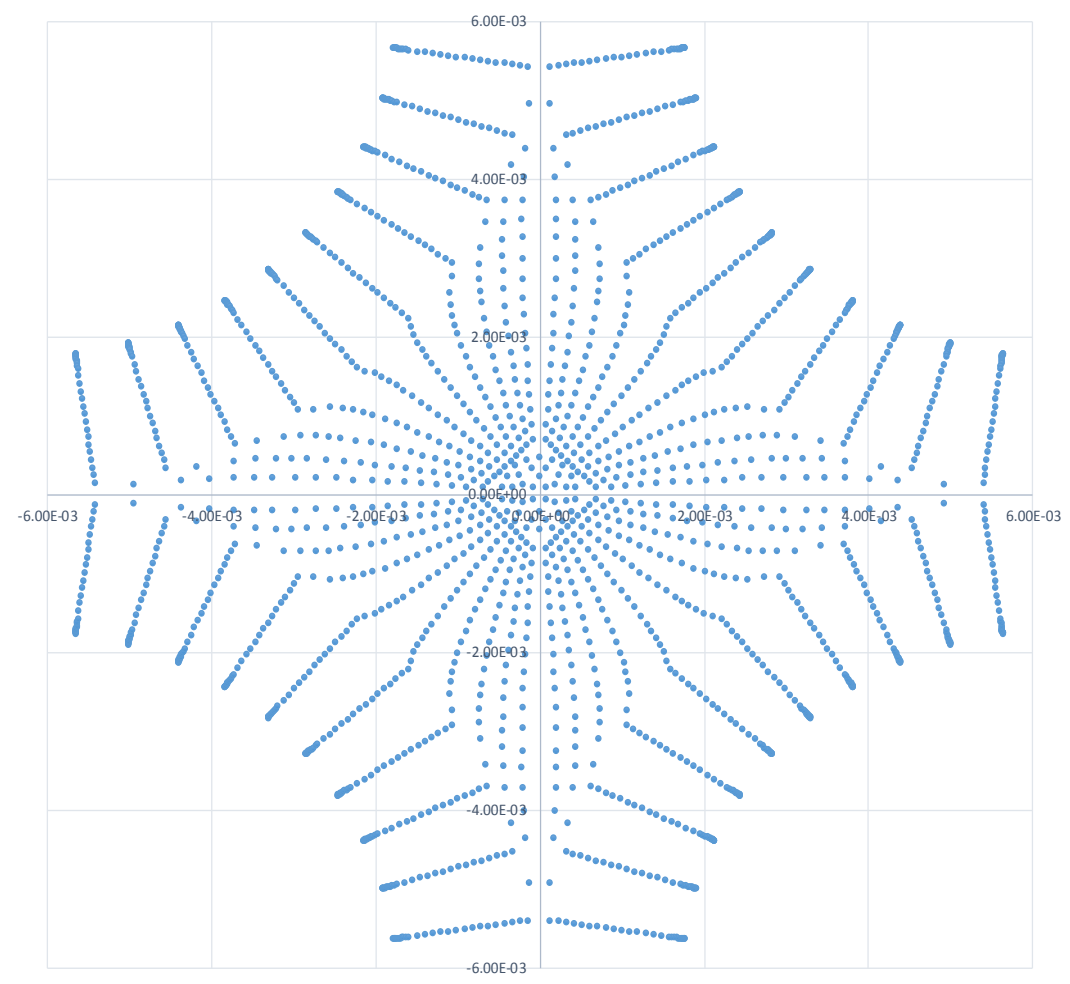

Fig 5. The virtual probe plane to record instantaneous turbulence quantities.

\subsection{Results and discussion}

The location of probe plane in case G1 is illustrated in Fig 4. To process the data, three time windows are chosen and each covers a time interval of about two flow-through periods. As shown in Fig 6 , the mean velocity profiles from three adjacent windows display a very good convergent trend, which indicates that the turbulent flow has reached statistically steady state regime.

One can non-dimensionalize the mean velocity and distance to the wall using the friction velocity $\left(u_{\tau}=0.037 \mathrm{~m} / \mathrm{s}\right)$ and wall length scale $\left(\delta_{l}=3.25 \times 10^{-6} \mathrm{~m}\right)$ respectively. The resultant velocity profile is plotted in a semi-log scale and can be compared with the well-known turbulent Law of the Wall [11]. As shown in Fig 7, in the viscous sublayer, the dimensionless velocity is approximately equal to the dimensionless wall distance. The second region is called buffer region. Following the buffer region, there is also a region where dimensionless velocity is proportional to the logarithm of the wall distance (i.e. $\left.U^{+}=\frac{1}{\kappa} \log y^{+}+B\right)$, referred as log-law region. The log-law fitting results in the model constants $\kappa$ of 0.42 and $B$ of 6.5 . 


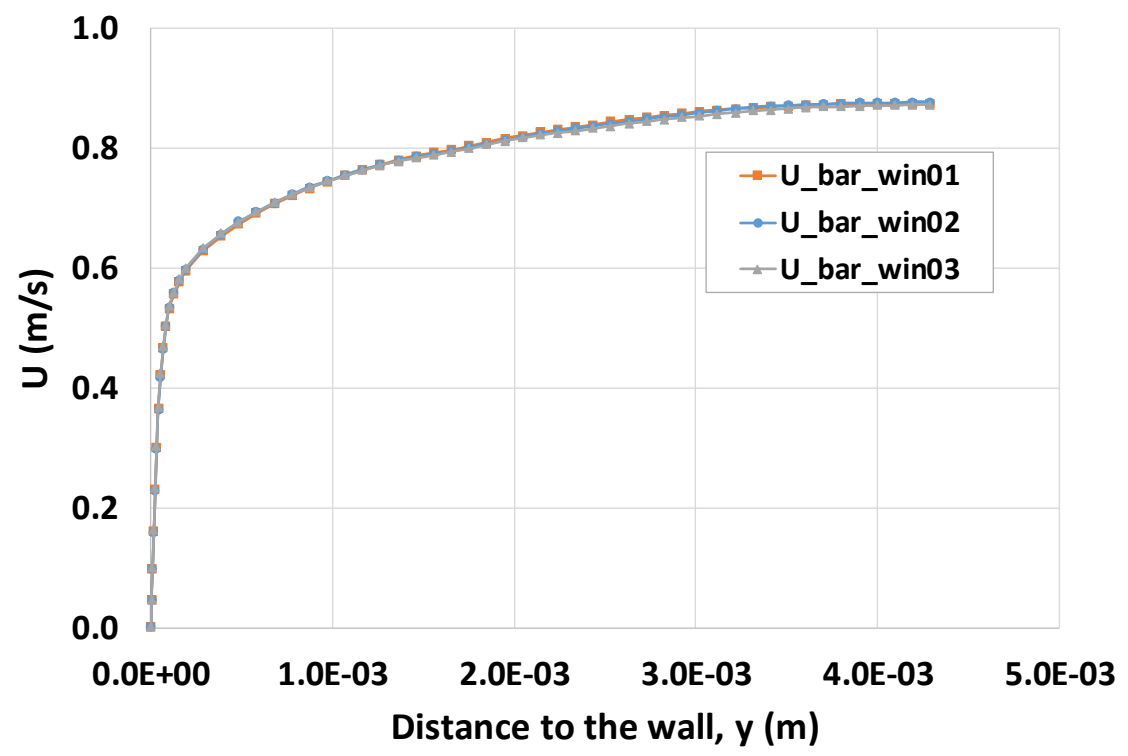

Fig 6. The mean velocity profile in case G1.

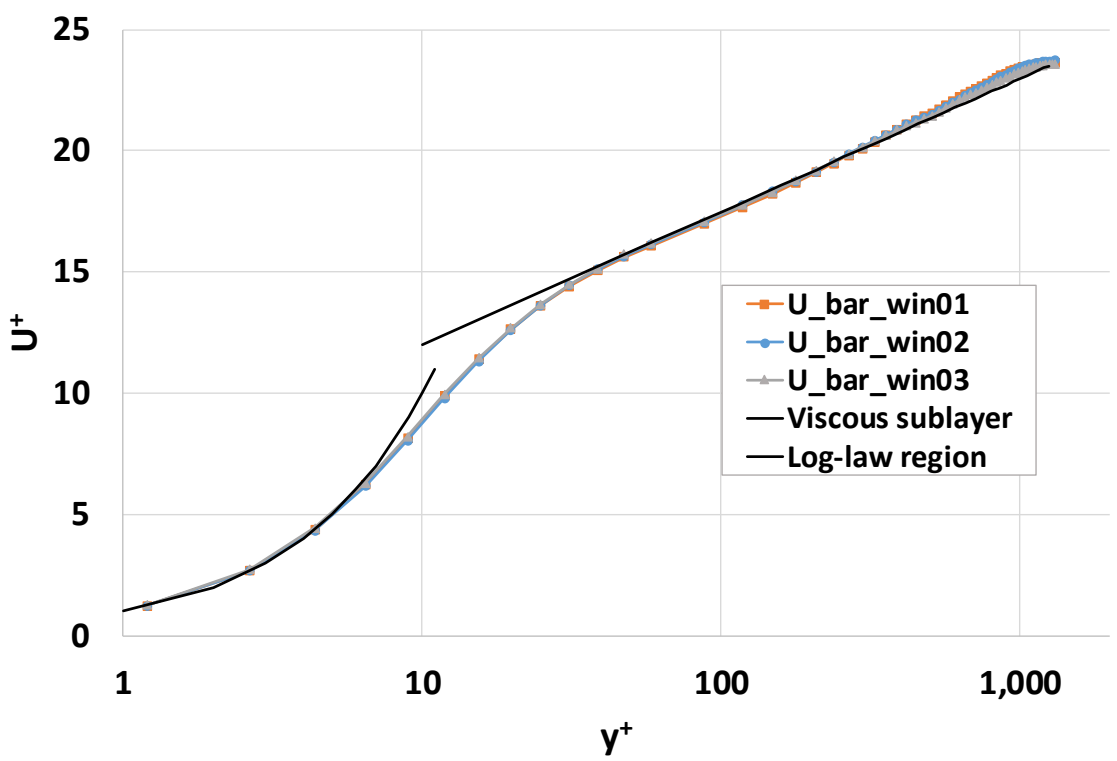

Fig 7. The non-dimensional mean flow velocity profile in case G1.

Addition of the fully resolved spacer grid and mixing vanes (SGMV) to the computational domain allows to analyze the turbulence in realistic engineering geometry. Fig 8 visually shows the impact of SGMV on the turbulence field as a lot of vortices are induced around the surfaces of SGMV. At a downstream location (1.3 cm from the endpoint of SGMV region), a probe plane is placed to record the turbulence flow field information. 


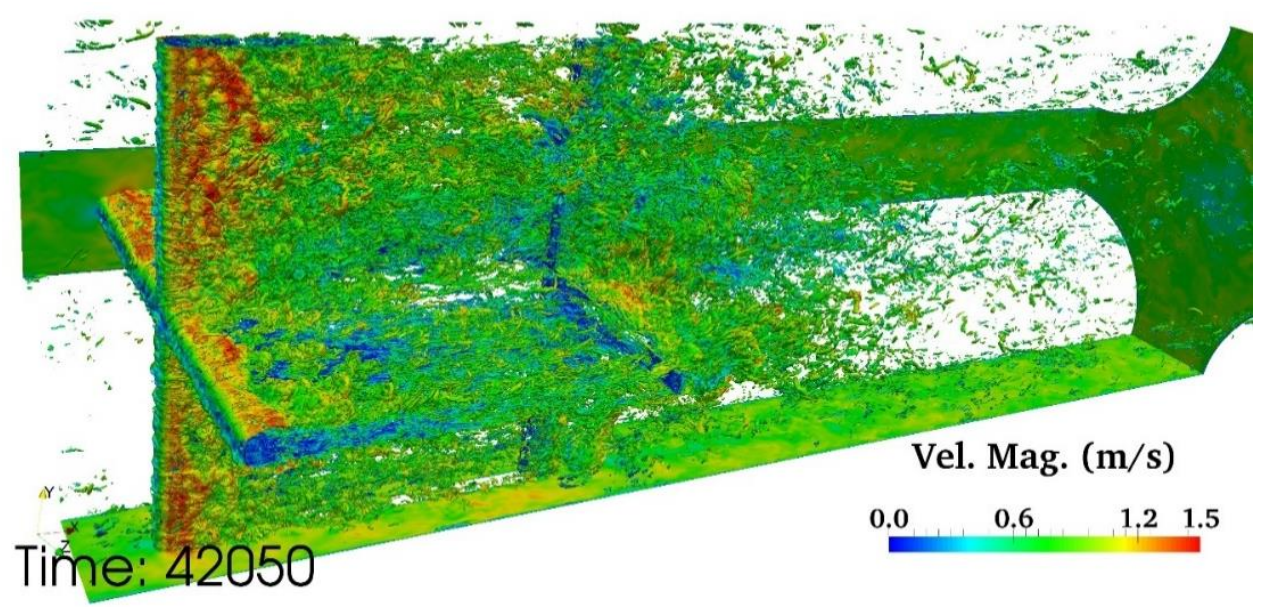

Fig 8. The turbulence vortices induced by the spacer grid and mixing vanes.

We have recorded the instantaneous velocity history for a time interval of five flow-through periods. The mean flow velocity profile obtained is shown in Fig 9. In contrast to the profiles shown in Fig 6, the magnitude of mean velocity in case $\mathrm{G} 2$ would drop after the initial rapid rise as the wall distance increases. This behavior can be explained by the existence of mixing vanes. The mixing vanes as geometric constraints would force the bulk flow to move towards peripheral region, and thus cause the drop of mean velocity magnitude at domain centerline. The dimensionless velocity profile is shown in Fig 10. If one assumes that the velocity profile in turbulence viscous sublayer is universal (i.e. $u^{+}=y^{+}$is valid), an estimated friction velocity can be determined, which is $u_{\tau}=0.052 \mathrm{~m} / \mathrm{s}, 40 \%$ larger than that in a regular subchannel geometry. This also indicates the wall stress in case $\mathrm{G} 2$ is about $40 \%$ larger than that in case G1. The log-law model constants in this case are $\kappa$ of 0.6 and $B$ of 7.6.

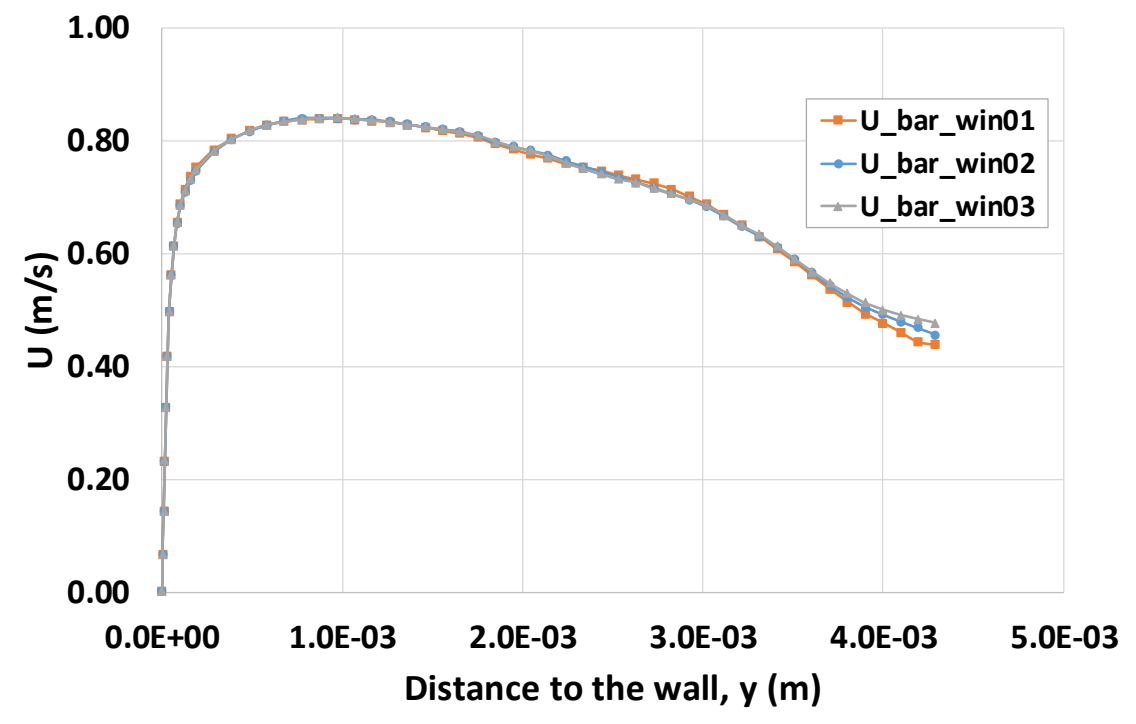

Fig 9. The mean velocity profile in case G2. 


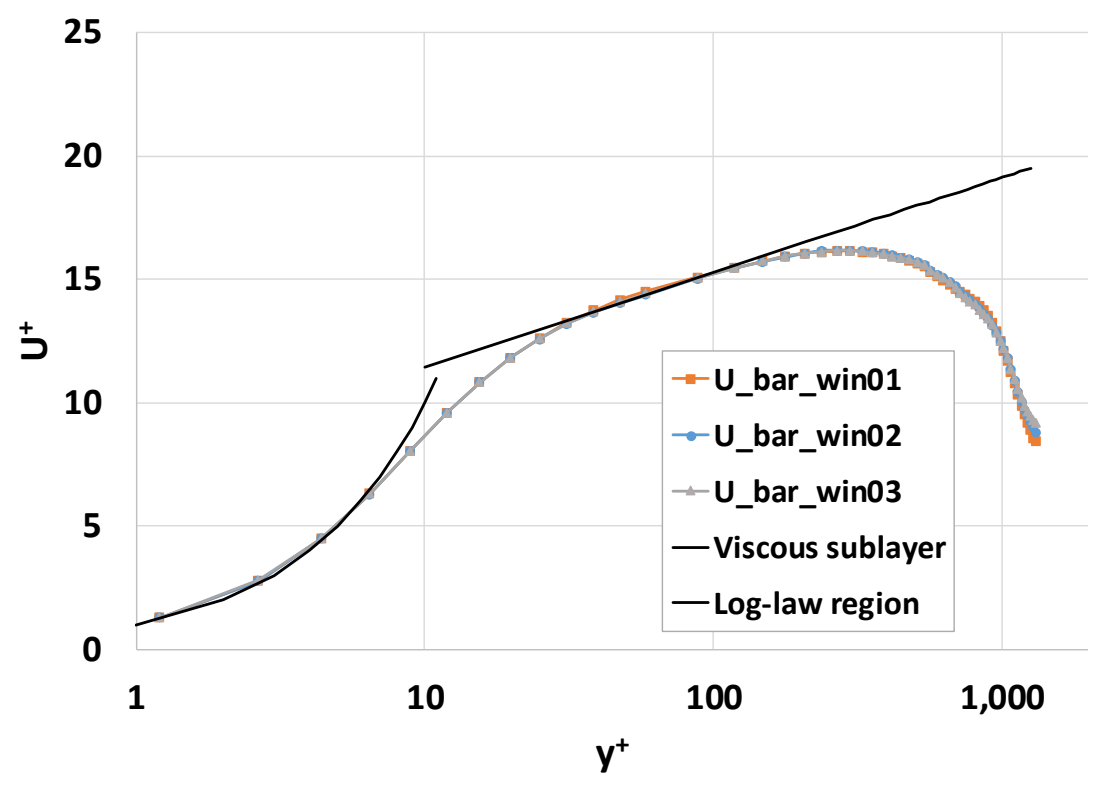

Fig 10 . The non-dimensional mean flow velocity profile in case G2.

In the domain G3, the extended channel height allows recording the turbulence field information at several different downstream locations. Four measurement planes (MP) are adopted in the domain G3, and they are labeled from MP1 to MP4 as described in Table 4. The instantaneous velocity field exhibits a centrifugal/rotational pattern due to the geometry constraints of mixing vanes (Fig 11). The comparison of mean flow velocity profiles is depicted in Fig 12 for different MP's as well as the free subchannel domain (G1) with no internal structure. The skewed mean flow velocity profile is observed at all MP's. The mean flow velocity tends to have a more flattened profile (outside the near wall region) when the MP location is increasingly far away from the SGMV. Fig 12 dynamically illustrates the decay of SGMV influence as the flow leaves the SGMV region.

We also obtained the turbulent kinetic energy (TKE) profiles for all measurement planes. The comparison is shown in Fig 13. Compared to the free subchannel (G1), the existence of SGMV dramatically increases the TKE magnitude in the coolant flow. The enhancement of coolant turbulence will help achieve higher convective heat transfer efficiency, but also requires larger pressure gradient to sustain the flow rate. A prominent TKE peak is observed at the wall distance of $3.0 \mathrm{~mm}$, of which the location is highlighted in Fig 14. The location of TKE peak is closely related to the height and orientation of mixing vanes. As the measurement location moves away from the endpoint of SGMV, the TKE magnitude will decay progressively due to the continued dissipation. 

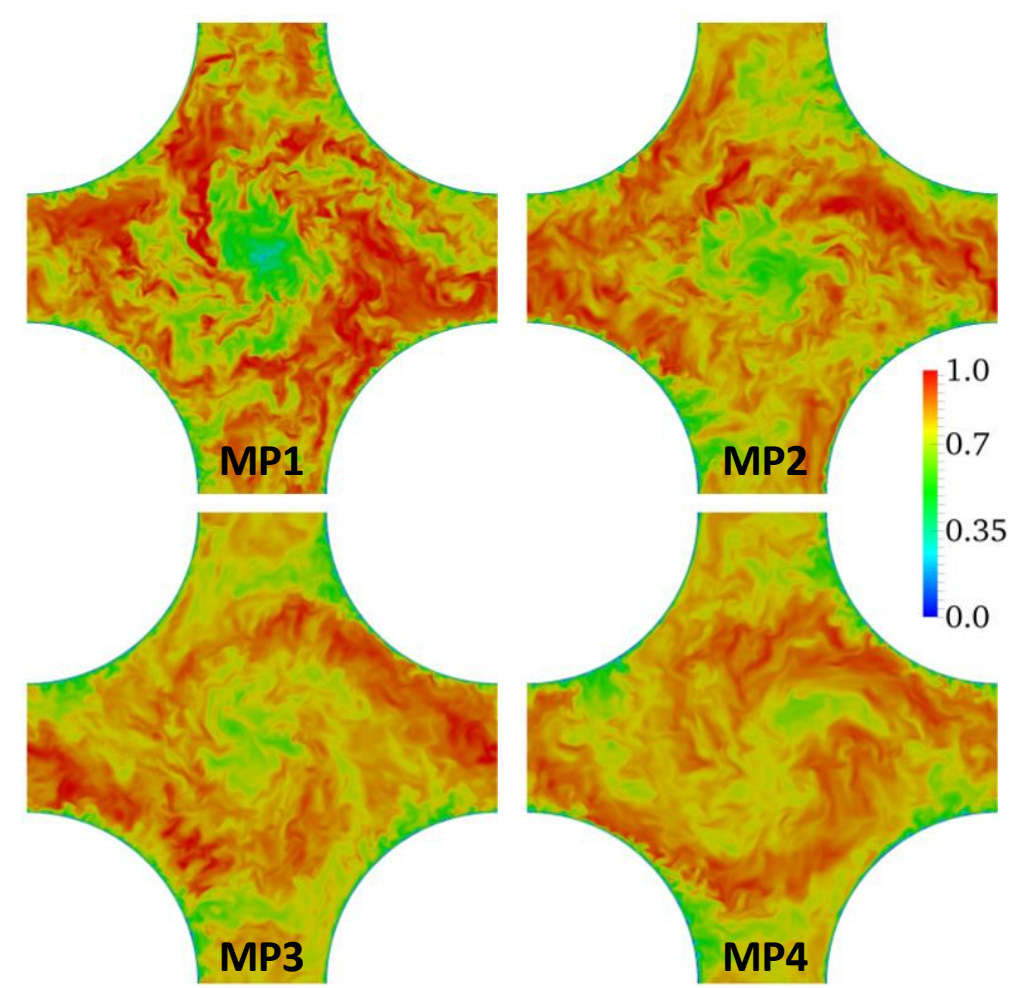

Fig 11. The instantaneous turbulence velocity field of the last time step at all measurement planes in domain G3.

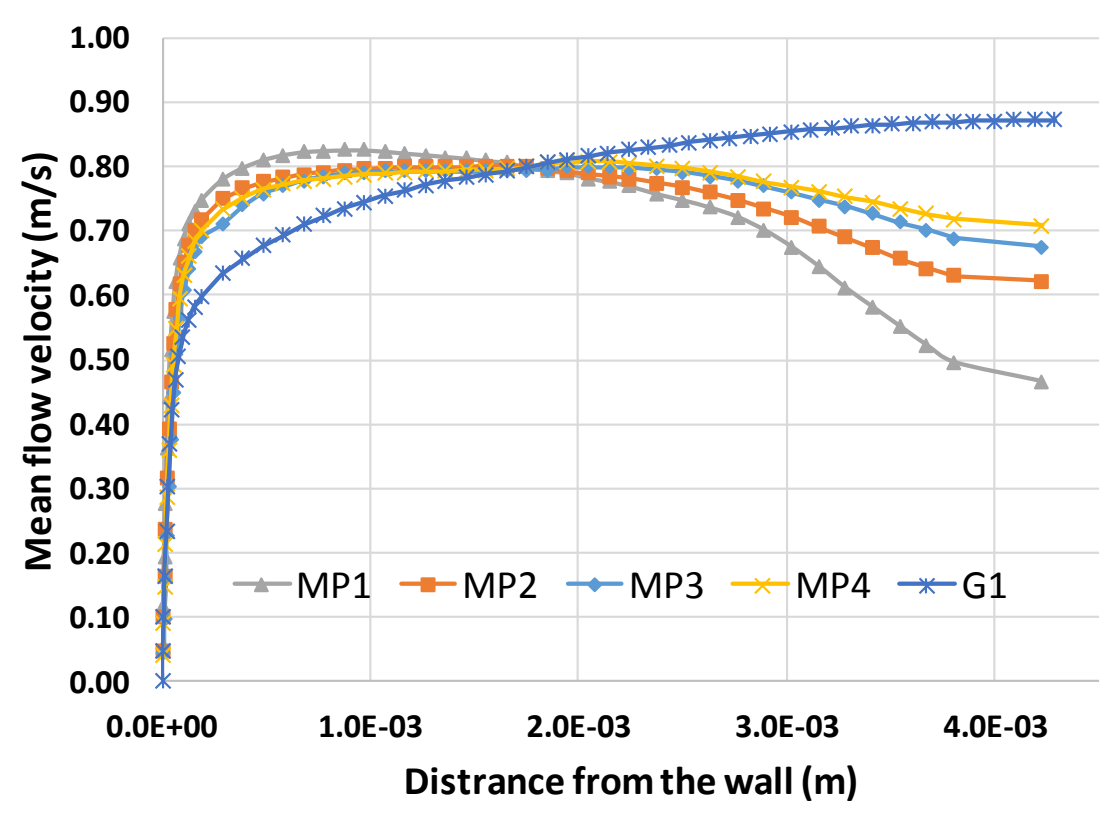

Fig 12. The mean flow velocity profiles at different downstream locations as well as the free subchannel (G1). 


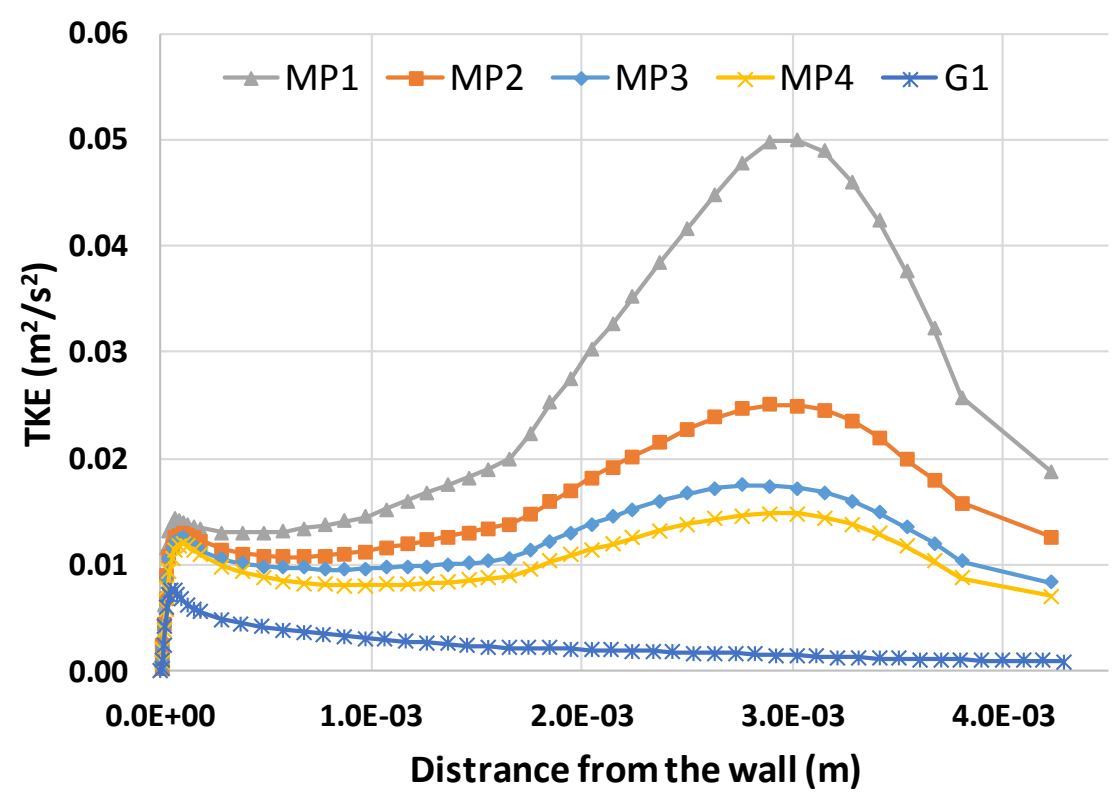

Fig 13. The turbulent kinetic energy profiles at different downstream locations as well as the free subchannel (G1).

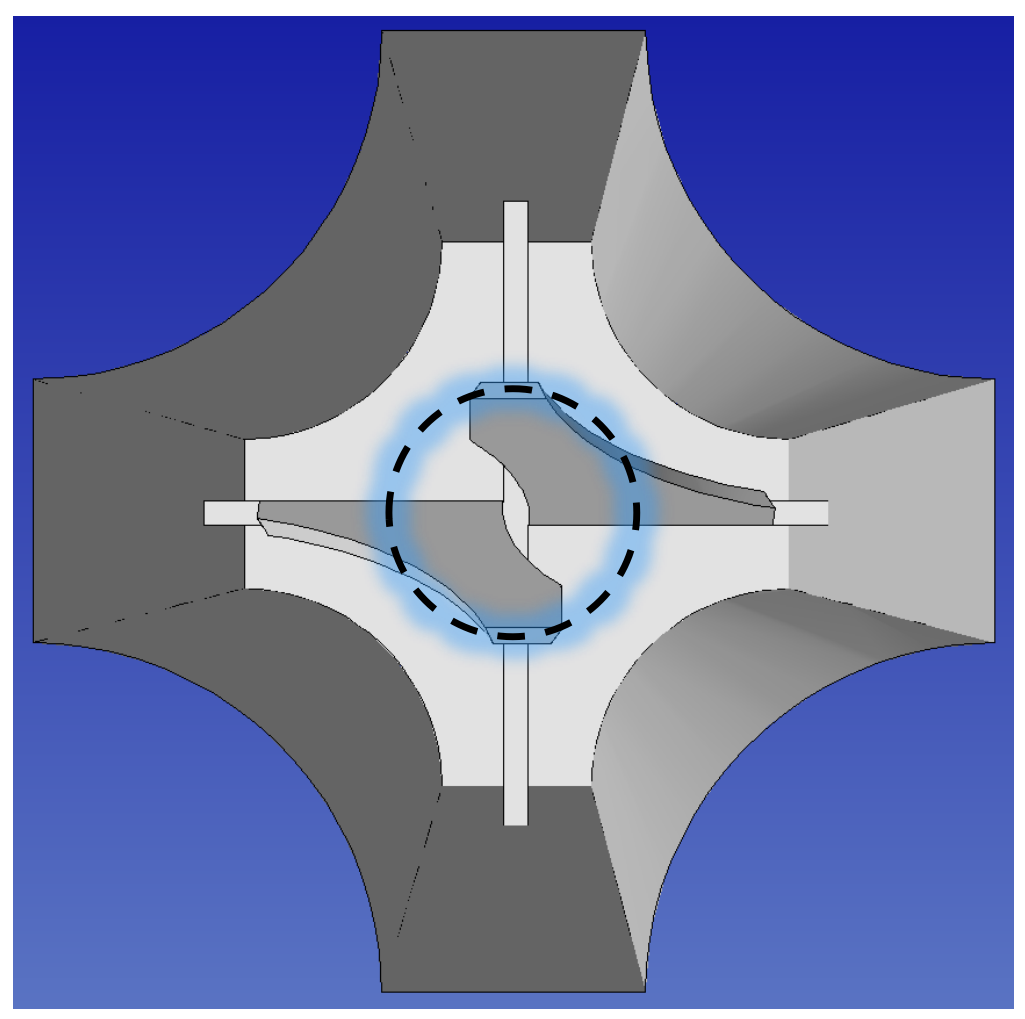

Fig 14. The highlighted location of observed TKE peak with respect to the 


\section{Using DNS to Examine Turbulent Bubbly Flow in Vertical Channels}

\subsection{DNS databases for bubbly flows}

DNS results provide any quantity at every spatial location and instance in time. It is therefore possible to compute any averages or other statistical quantities desired directly. While we have computed a number of such quantities of interest for several different runs, we have only collected relatively conventional ones and many others are of interest to investigators pursuing averaged or reduced order models of the flow. To facilitate access to our data for investigations by other researchers we have generated a database of fully resolved flow that we are making available to everybody who wants to use it. In order to keep the flow simple but relevant to realistic flows and modelers we have selected a case with deformable bubbles that do not collect at the walls to form prominent wall-layers. As of now we prefer to work with clean bubbles, since surfactants would add one more governing number and both the amount of surfactant and their exact constitutive equations are often unknown. While surfactants can dramatically change the flow structure of flow with nearly spherical bubbles (as documented in a manuscript submitted for publication) surfactants are likely to have smaller effect on deformable bubbles (see below). The wall layers formed by nearly spherical clean bubbles are rarely seen experimentally, either because such bubbles are almost always affected by the presence of surfactants or because small bubbles collecting at walls coalesce into larger bubbles that are not driven towards walls. In the current study we do not allow coalescence and use only bubbles of one size, to simplify the statistical description.

The parameters governing the setup are listed in Table 5 and in Fig 15 we show the bubbles and the vorticity, visualized by the lambda-2 method, after the flow has reached a steady state. The average evolution is shown in Fig 16 where we plot the flow rate and the wall shear. At steady state the average wall shear should be equal to the pressure gradient and weight of the mixture, shown by the dashed line. Although there are significant fluctuations, due to the relatively small size of the domain, the average is close to the dashed line. The volume flow initially decreases slightly but then levels out. Fig 17 shows several quantities averaged over planes parallel to the walls, versus the wall normal coordinate. The figures are plotted with data from time $=500$ to time $=774$, after the bubbly flow reaches an approximately statistically steady state. The first two frames show the liquid velocity (left) and the void fraction (right). Both are slightly asymmetric, indicating that we need longer time for the averages to fully converge. The velocity profile is similar to what we see for single-phase flow and the flow rate, as seen in Fig 16, is nearly the same. The void fraction profile shows that the bubbles remain distributed over the core of the channel and that the region next to the walls is essentially free of bubbles. We expect the average void fraction distribution to eventually exhibit a smoother shape, once the statistics has converged fully. The next two frames show the root mean square of the velocity fluctuations of the three components (left) and the $\left\langle u^{\prime} v^{\prime}>\right.$ Reynolds stress component. The stream wise velocity fluctuations are largest and peaked at the wall, and the anisotropic Reynolds stress component shows the nearly linear behavior in the center of the channel typically seen in single phase turbulent channel flows (and unlike what is seen for nearly spherical bubbles forming wall layers, where it is close 

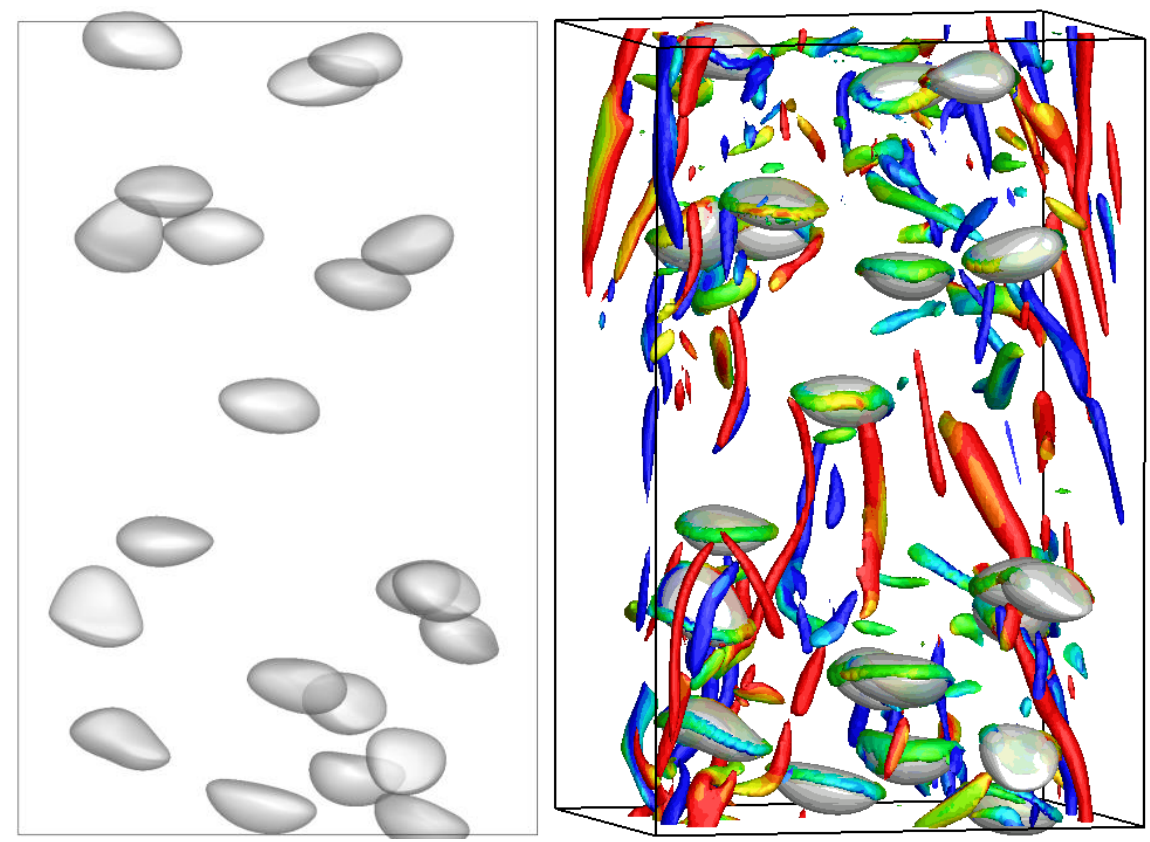

Fig 15. Showing the bubbles (left) and the bubbles and iso-surfaces of lambda-2 $=-1.5$ at time 500 (right).
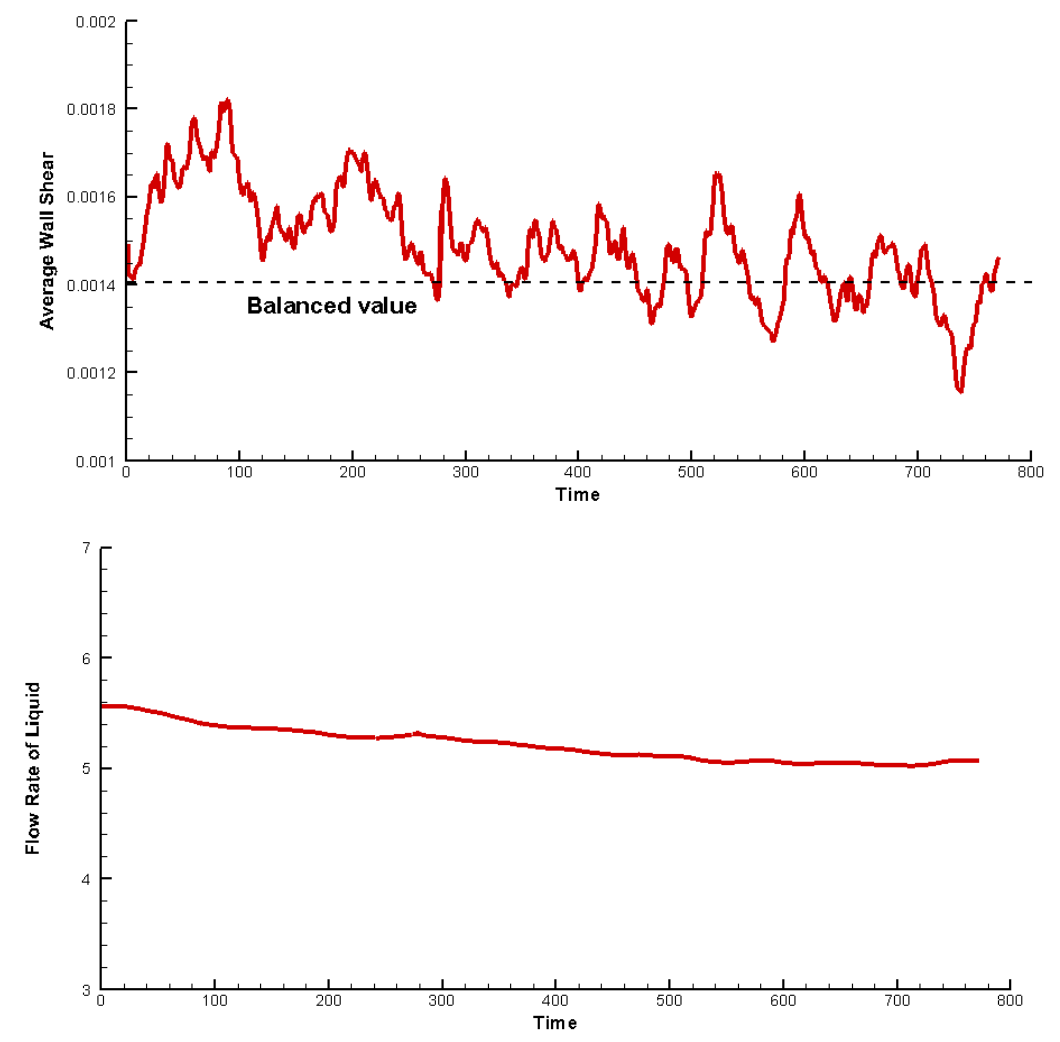

Fig 16. The average wall shear (top) and the flow rate (bottom) versus time. 

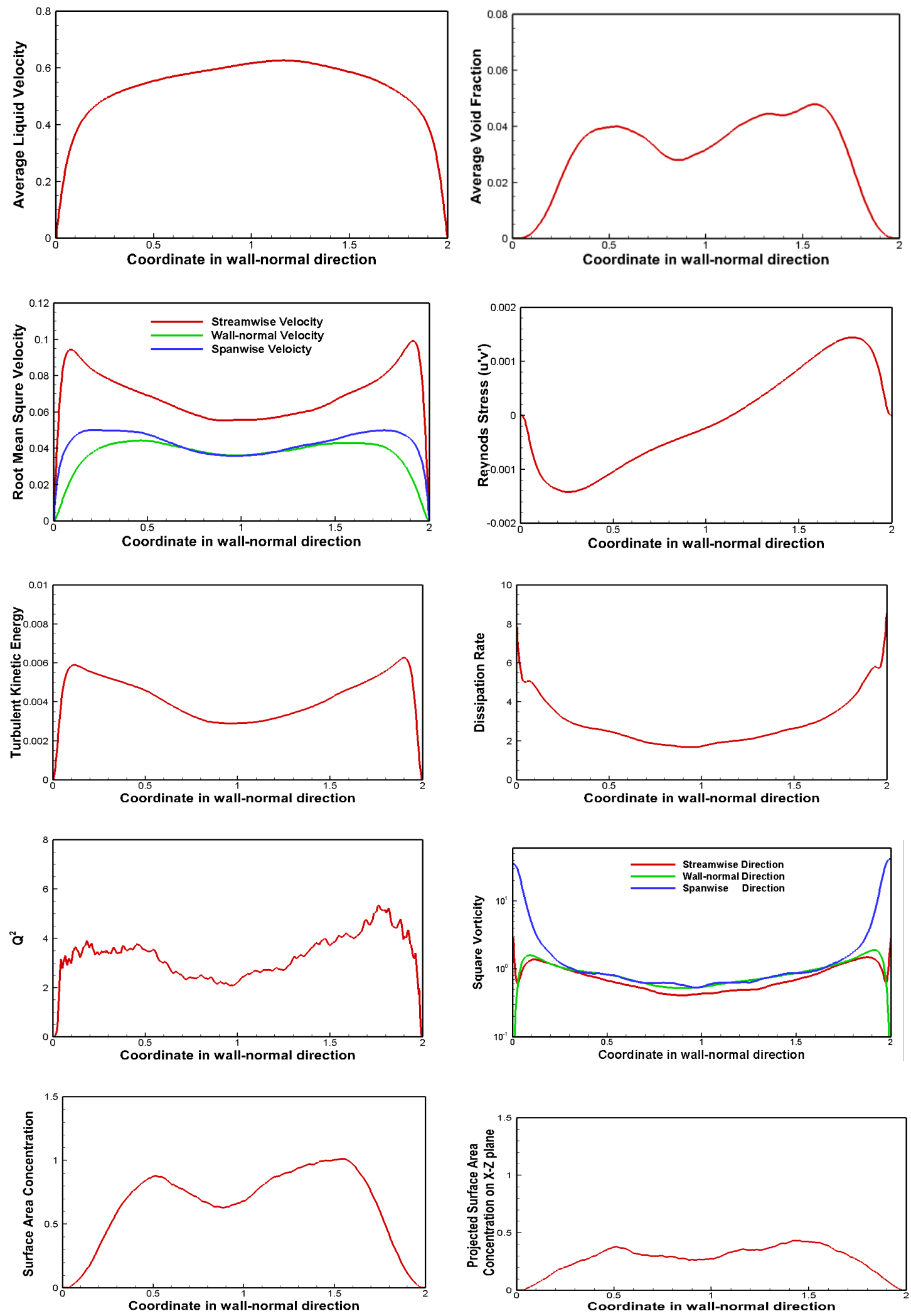

Fig 17. Several quantities averaged over planes parallel to the walls, versus the wall normal coordinate. 
to zero. The turbulent kinetic energy (left) and the turbulent dissipation rate (right) also show behavior reminiscent of single-phase flows. The next pair of frames show the magnitude of the second invariant of the velocity in the liquid (left) and the vorticity squared (right). As expected the spanwise vorticity (parallel to the wall and perpendicular to the flow) is largest near the walls where the shear is largest. The second invariant of the velocity in the liquid is found by

$$
Q_{2}=\frac{u_{i}}{x_{j}} \frac{u_{j}}{x_{i}}
$$

and is often used for visualizing the vertical structures, since it gives similar results as the lambda-2 method. Finally, the bottom two frames show the average area density and the projection of the area in the streamwise direction. We are currently continuing the run, gathering additional statistics. We are, in particular, hoping that the averages will become more symmetric, as they should as the time becomes longer. If the required time becomes excessively long we can, however, always average the results over the left and the right-hand side of the domain.

\subsection{Effect of surfactants on deformable bubbles}

As mentioned above, we have examined in some detail the effect of insoluble surfactant on the collective motion of nearly spherical bubbles and showed that the surfactant has significant impact on the structure of the flow. The bubble rich wall layers and the significant flow reduction (for constant imposed pressure) seen for clean bubbles is, in particular, not seen when surfactant is present. This study was described in a draft included with last year's final report and has now been submitted for publication. We have also conducted a preliminary study of the effect of surfactant on deformable bubbles and found that the impact is much smaller than for nearly spherical bubbles-as expected. Fig 18 shows the bubble distribution from a simulation of clean bubbles (left frame) and a simulation of bubbles with surfactants (right frame). The parameters used are listed in table II. Obviously there is not an obvious difference between the average bubble distributions in the two frames. The flow rate and the wall shear versus time are plotted in Fig 19. Here it is clear that we see neither the large increase in wall nor the reduction in flow rate usually seen for clean nearly spherical bubbles. Indeed,

Table 5: Case setup parameters

Domain size: $\pi \times 2 \times 0.5 \pi(x, y$, and $z)$

Resolution: $384 \times 256 \times 192$

Frictional Reynolds Number: 150

21 Bubbles with diameter of 0.3

Average void fraction: $3 \%$

Surface Tension Coefficient: 0.00225

Density (fluid/bubble) : 1.0/0.1

Viscosity (flulid/bubble) : $2.5 \times 10-4 / 2.5 \times 10-4$

Gravity (gx): 0.1

Eotvos Number: 4.0
Domain size: $\pi \times 2 \times 0.5 \pi(x, y$, and $z)$

Resolution: $256 \times 192 \times 128$

Frictional Reynolds Number: 127.3;

Initially 24 bubbles of diameter of 0.30

Average void fraction $5.94 \%$

Tension Coefficient: 0.0016

Density: 1.0/0.05

Viscosity: $3.33 e-4 / 3.33 e-5$

Gravity: 0.05

Spherical Bubbles: Eo $=2.81, \mathrm{M}=1.51 \times 10^{-7}$ 


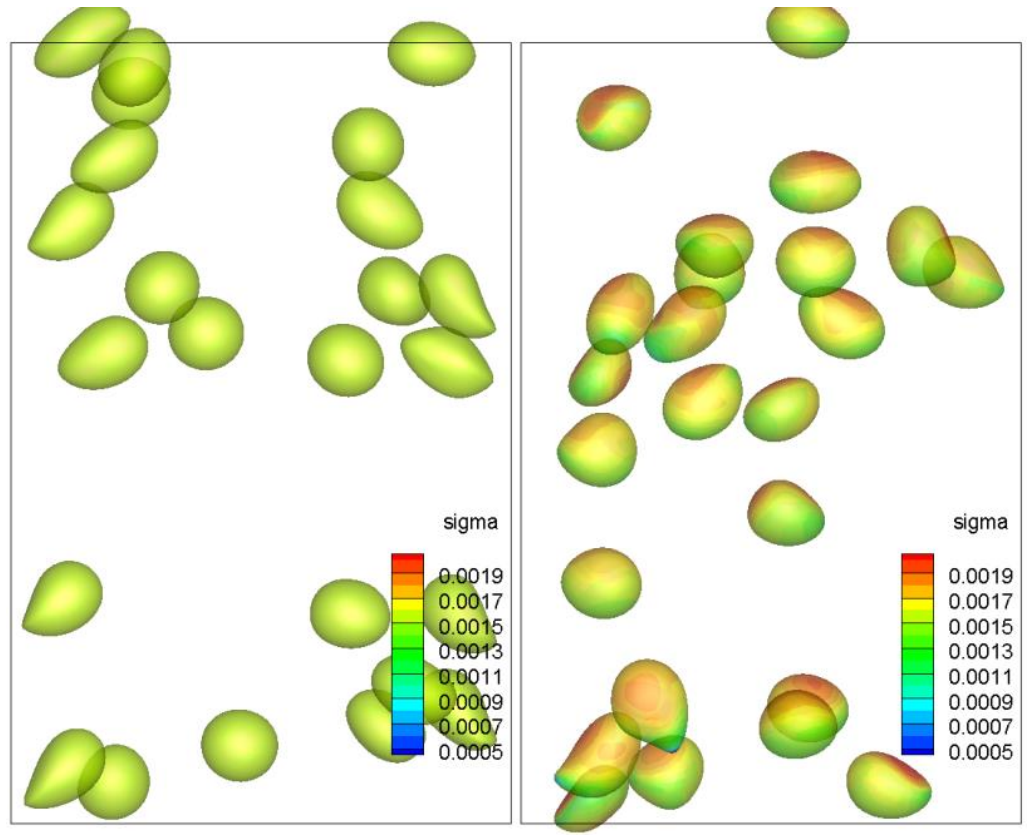

Fig 18. Two frames from simulations of deformable bubbles. In the left frame the surface tension is constant, but in the right frame it is a function of the concentration of insoluble surfactant.
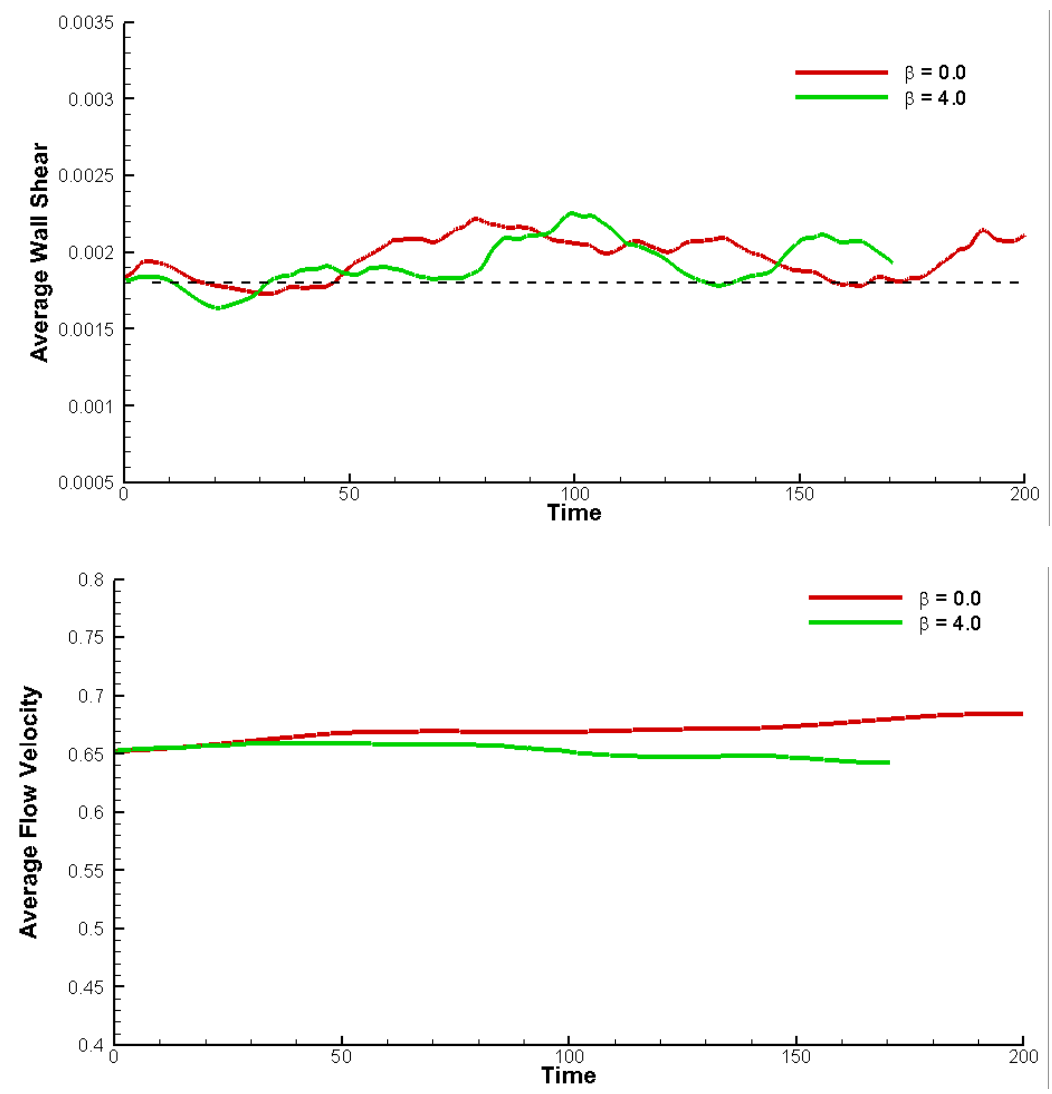

Fig 19. The average wall shear (top) and the flow rate (bottom) versus time. 
the curves for both the clean and contaminated deformable bubbles show that neither the flow rate nor the wall shear changes much. The similarity between the clean and contaminated deformable bubbles is the reason we have chosen to use deformable bubbles for the database described above.

\subsection{Advanced data processing}

For single-phase turbulent flows major progress has been made in the analysis of the flow structure and its dynamics. Relatively little of these advances have been used for multiphase flows, in part since it is only recently that DNS data for multiphase flows, of the quality available for single phase flows, has become available and also due to the greater complexity of multiphase flows. In addition to progress in analyzing single-phase flows, advances are also being made in characterizing heterogeneous media. In most cases those are, however, stationary or undergoing only limited deformation. Multiphase flows where a sharp interface separates different fluids evolving in a complex turbulent flow combine the challenges of turbulent flows and heterogeneous media.

We have described our efforts to use machine (or statistical) learning to help us develop simple closure relationships in earlier reports. Our early efforts focused on laminar bubbly flows (and were in part supported under a grant from the National Science Foundation). In those cases we found that the closure terms could be represented as functions of the averaged quantities themselves. We are now focusing on turbulent flows, which are of more relevance for realistic flows, and for such flows the closure terms in equations for the average flow usually rely on quantities describing the average state of the unresolved scales. In single-phase flow such quantities include the turbulent kinetic energy and the dissipation rate, but other choices are possible. In multiphase flows there are more such quantities and we are examining the use of "feature selection" method to help us determine which of those quantities correlate best with the closure terms. This effort is still in its early stages and we expect to have more complete picture by the end of the year. We note that once such quantities have been identified, we still need to determine how to find them and that we are likely to do so with evolution equations that contain closure terms of their own.

Most recently we have been examining how the data produced by the simulations can be reduced using various decomposition methods, where we attempt to map the dynamics onto a few energetic modes. We started by studying Proper Orthogonal Decomposition (POD), where the various quantities are mapped onto an optimal linear basis. This decomposition is by now used in very diverse fields, under several different names. As Singular Value Decomposition it does, for example, play a major role in various machine-learning applications. POD was introduced into studies of turbulent flow in [12] but continues to be used and developed. The availability of large-scale DNS results has, in particular, provided a growing impetus for widespread use. We are not aware of extensive use of POD for DNS of multiphase flows, probably because DNS results comparable for those for single-phase flows have only recently become available. We note that our exploration of the use of POD is still in a very early stage. Several extensions of POD have been proposed and while we expect to study the applicability of those for our problems, we have not done so yet. 

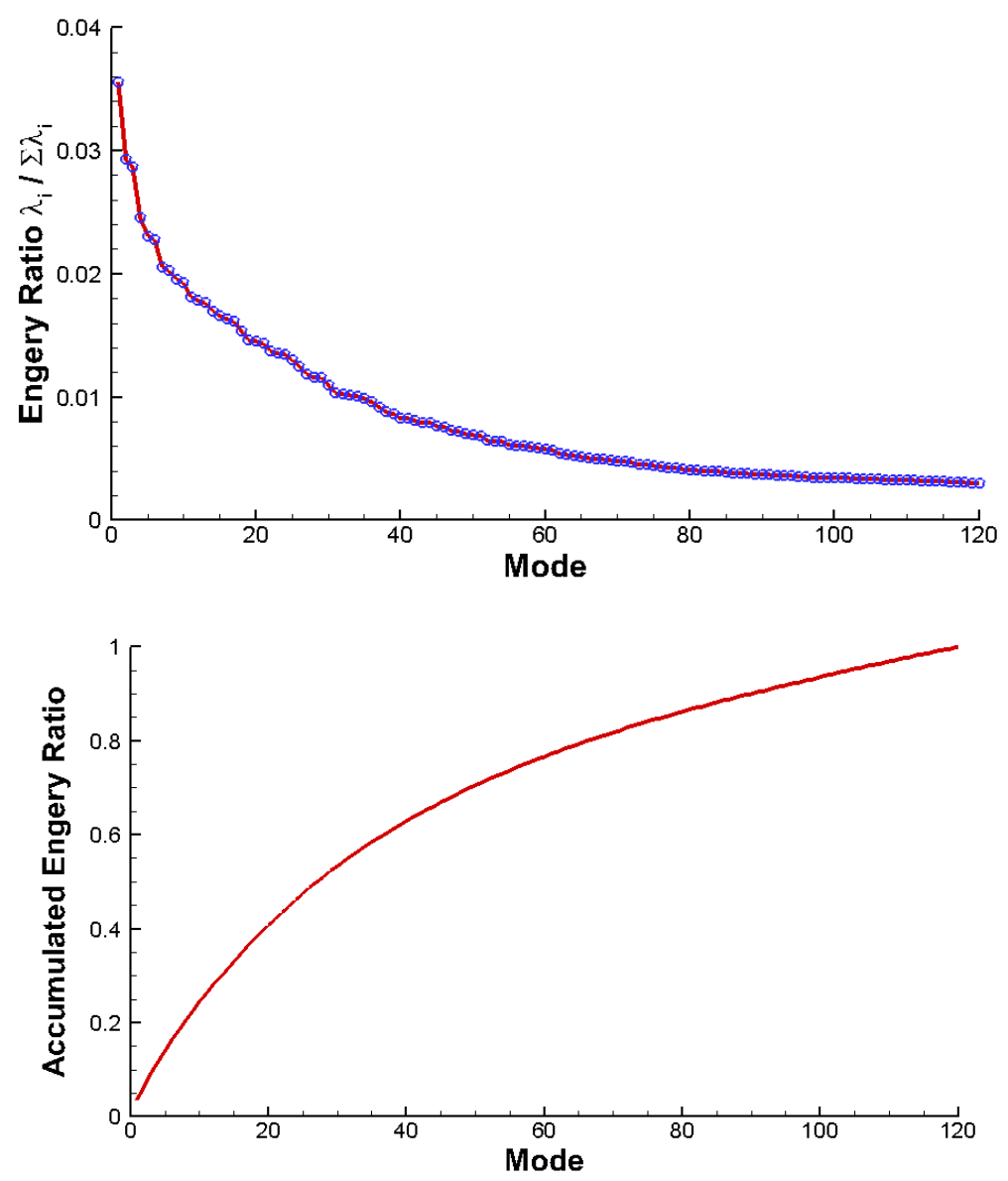

Fig 20. The energy of the modes for POD. The top frame shows the energy ratio (the energy in each mode divided by the total energy) and the bottom frame shows the accumulated energy versus the mode number, arranged in order of decreasing energy.
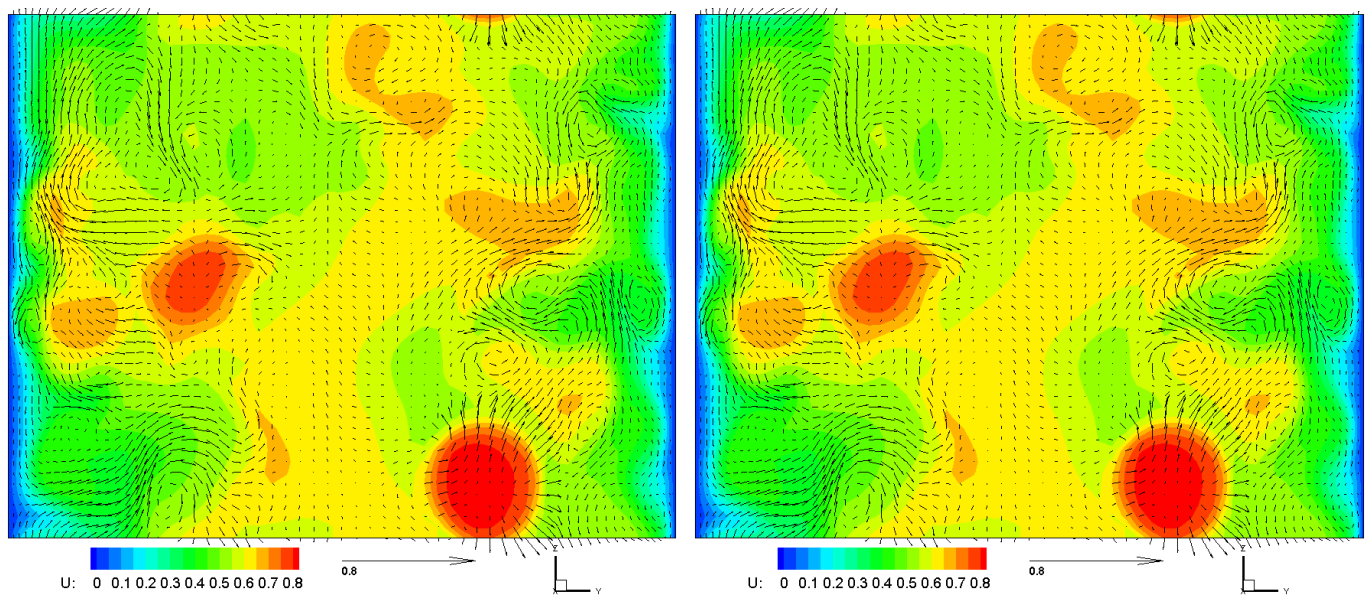

Fig 21. Original data (left). Reconstruction using 120 POD modes (right). 

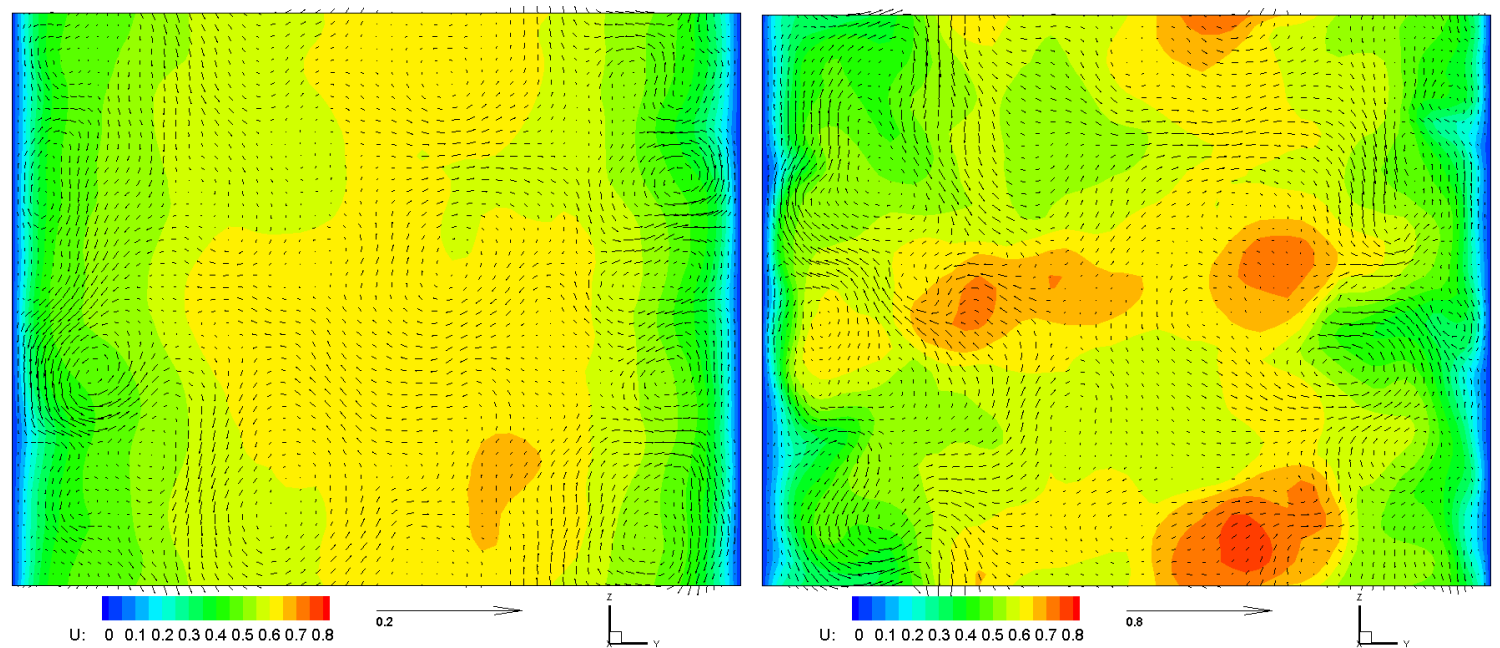

Fig 22. Reconstruction using the first POD mode only (left). Reconstruction using twenty POD modes (right).

To examine the use of POD for bubbly channel flows, we have implemented the Tall and Skinny QR decomposition method from reference [13] to handle the very large datasets that we are working with and then used LAPACK to find the POD modes. Fig 20 shows the energy content of the modes for the steady state portion of the flow in Figures 15-17. The top frame shows the energy ratio (the energy in each mode divided by the total energy) and the bottom frame shows the accumulated energy versus the mode number, arranged in order of decreasing energy. While the first few modes clearly contain significantly larger energy than the higher number nodes, it is also clear that the decay is relatively slow.

Fig 21 shows the velocity field in one cross section, perpendicular to the flow, at one time $(x=1.6$ and time $=766$ ). The left frame shows the original DNS data, the next frame shows a reconstruction using all the modes. As expected, those are essentially identical. In Fig 22 we show the flow field reconstructed by one mode (left), which is clearly completely inadequate, and using twenty modes. Although the flow field is starting to show similarity with the original data, it is clear that much information is still missing. We are currently examining the various extensions for the standard POD approach and if some of them will give us better concentration of the energy, so fewer modes suffice to describe the flow. If we are successful in doing so, the next step is to examine if the most energetic modes can be used in a reduced order model for the dynamics, probably using a Galerkin method to project the governing equations onto those modes.

Another approach, recently gaining in popularity, is the Dynamic Mode Decomposition (DMD), where the modes are no longer orthogonal. We have implemented the standard DMD described the same reference, but also experimented with the sparsity promoting DMD described in reference [14], using MATLAB codes downloaded from the authors website. We use 120 sequences of the whole turbulent velocity field to form the snapshot matrices, then apply the standard DMD. The resulting eigenvalues $\left(\mu_{\mathrm{i}}\right)$ are shown in Fig 23 and the dependence of the absolute value of the DMD amplitudes $\left(\alpha_{i}\right)$ on the frequency and the real part of the corresponding DMD eigenvalues are shown in Fig 24 . In order to select the reduced number of modes for a better approximation of the velocity fields, the 
sparsity-promoting method, described in reference [14], is applied to the standard DMD results. Fig 25 illustrates the optimal performance loss of the sparsity-promoting DMD in terms of the number of selected modes. The performance loss is quantified by the Frobenius norm of the approximation error between the reduced representation and the full data sequence in fraction of the Frobenius norm of the full data sequence. The performance is improved as the number of selected modes increased. However, it shows that the performance would not be perfect even if only one mode is missing. We will try to increase the number of the snapshots to get more modes in the future. The eigenvalues of the selected modes using the sparsity-promoting DMD are shown with the red circles in Fig 26 . The left shows that 4 modes are selected, while the right figure is the result for 22 selected modes. The relationships of the frequency with the amplitudes for the selected modes are drawn in Fig 27. It shows that the most modes selected by the sparsity-promoting DMD are the low-frequency ones, and they are not decided by the values of their amplitudes.

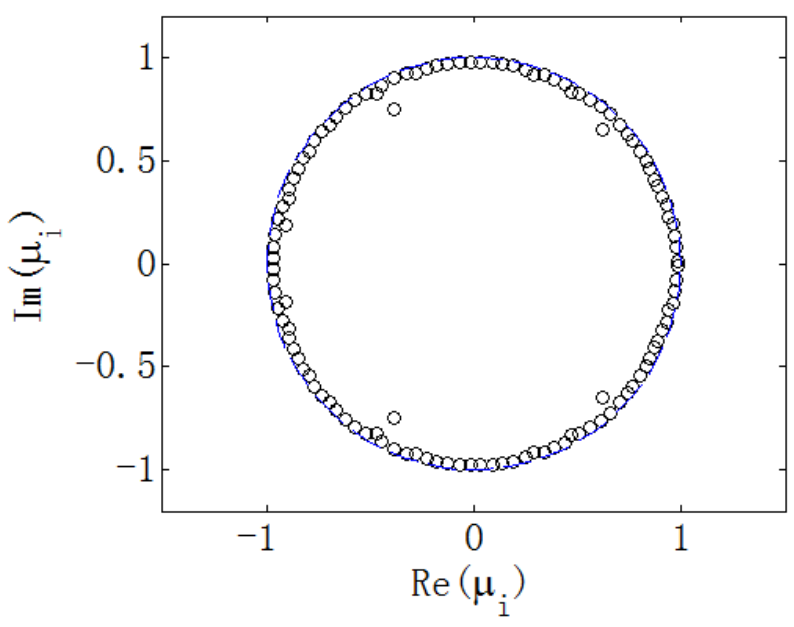

Fig 23. Distribution of Eigenvalues $\mu_{1}$ of all dynamic modes, as computed by the standard DMD.
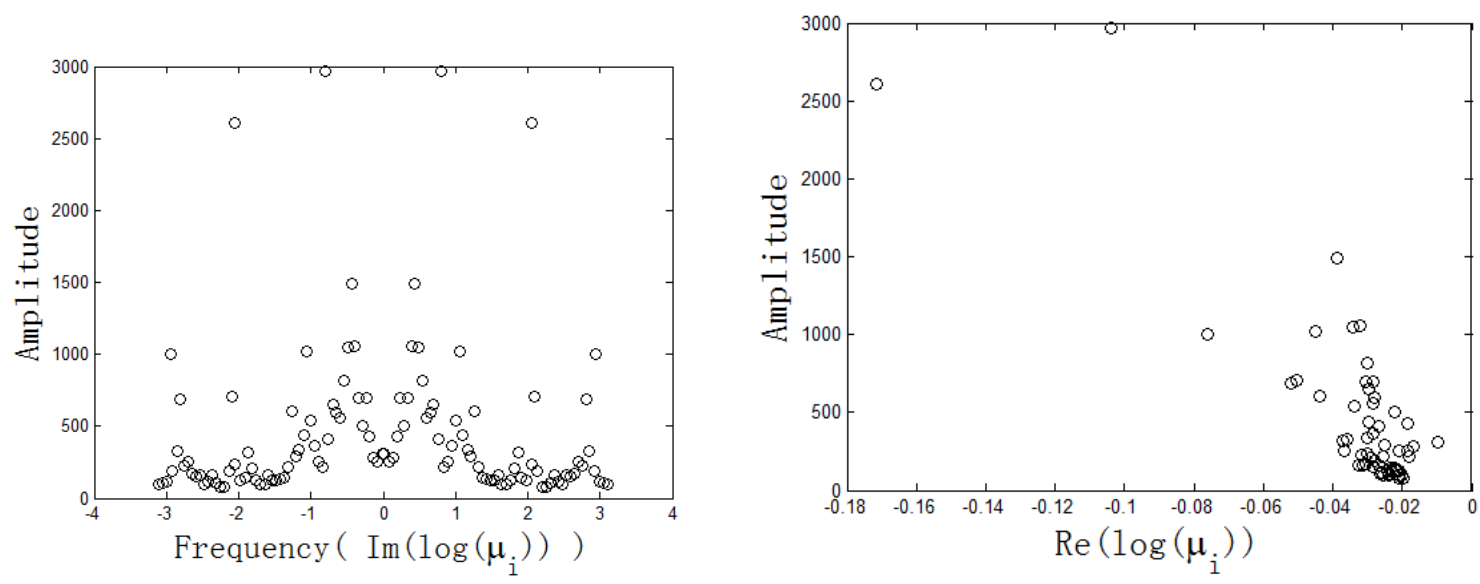

Fig 24. Dependence of the DMD amplitudes on the spectra (Left) and the real part (Right) of the corresponding DMD eigenvalues, as computed by the standard DMD. 


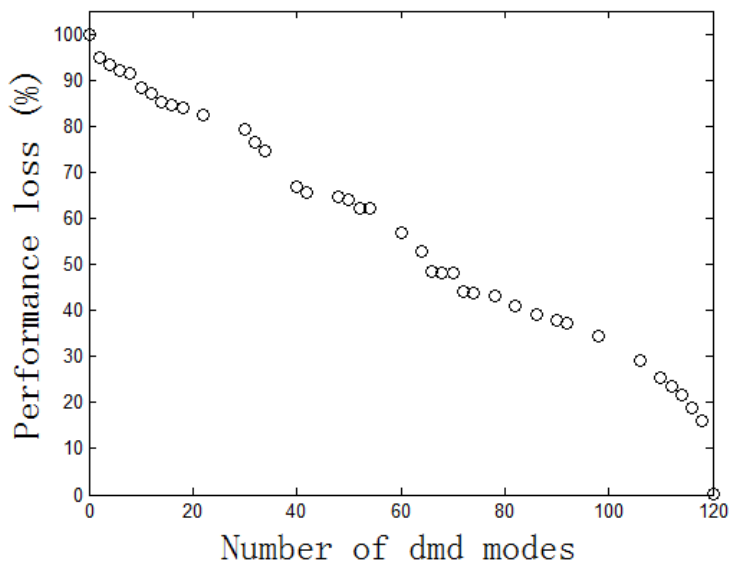

Fig 25. The optimal performance loss of the sparsity-promoting DMD
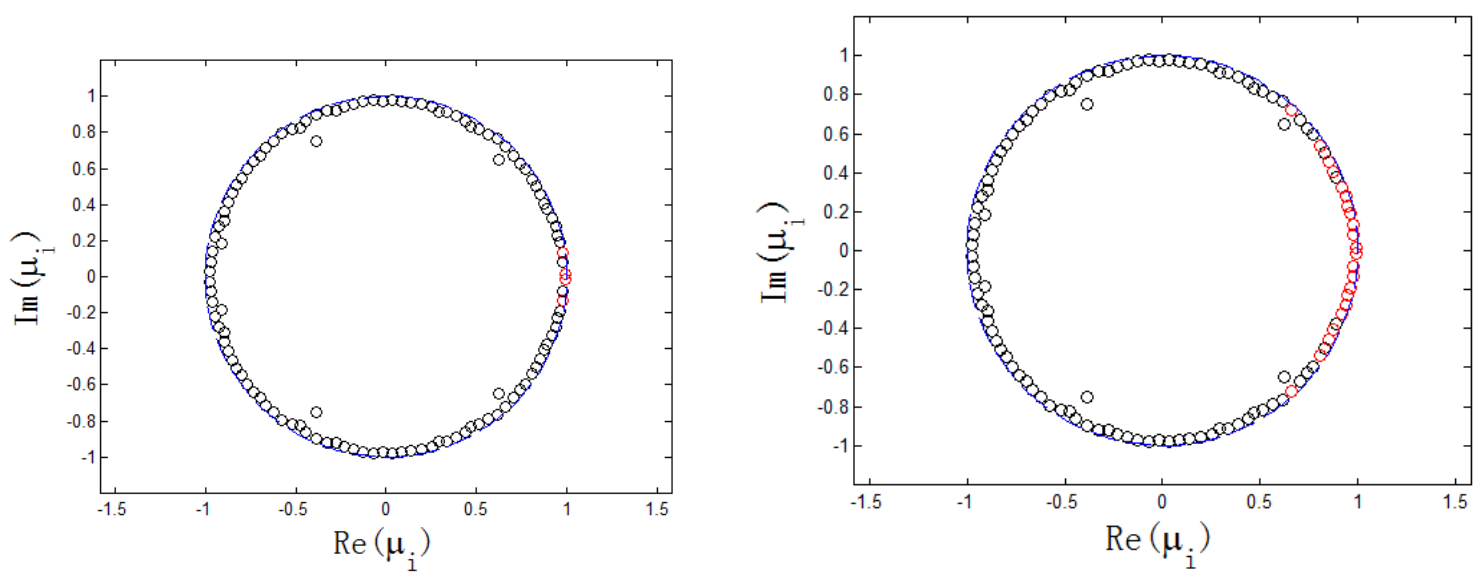

Fig 26. Eigenvalues (red circles) from the subset of $\mathrm{N}$ modes selected by the sparsity-promoting DMD algorithm. ( $\mathrm{N}=22$ for the top, and $\mathrm{N}=4$ for the bottom)
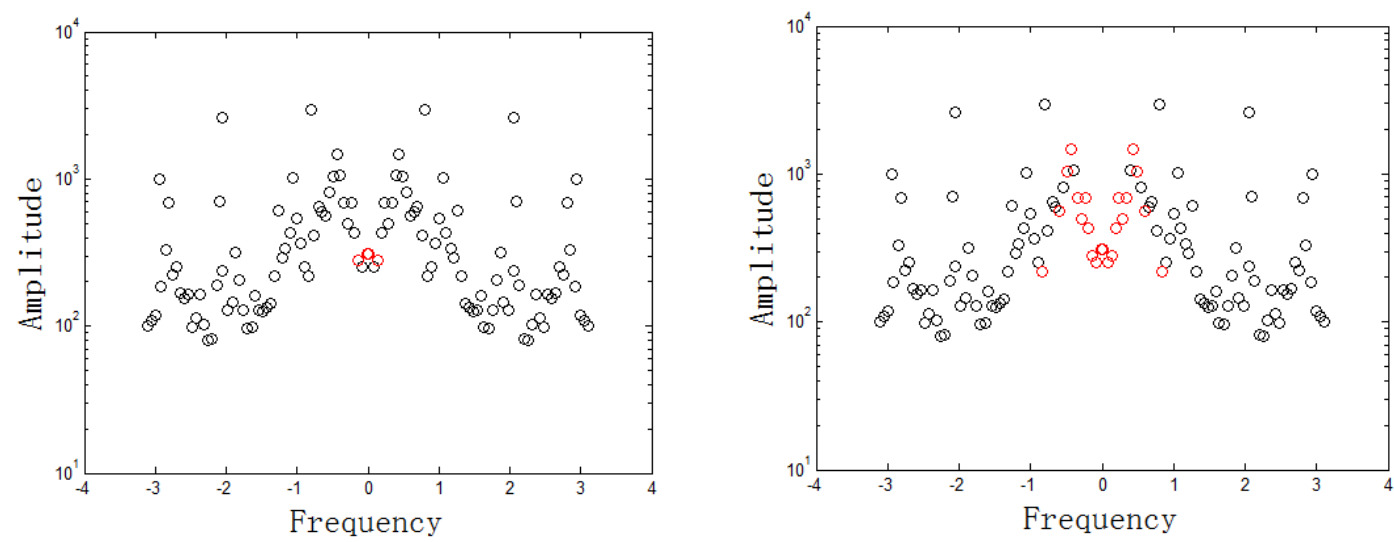

Fig 27. Dependence the amplitudes on the frequency for the subset of $\mathrm{N}$ modes selected by the sparsity-promoting DMD algorithm (red circles) with $\mathrm{N}=4$ for the left and $\mathrm{N}=22$ for the right. 


\subsection{Publications in preparation}

We continue to prepare our earlier results for publication. We have one paper that has been submitted and two in preparation. Drafts of all three were included with the final repost last year. The manuscript furthest along is:

J. Lu, M. Muradoglu, G. Tryggvason Effect of Insoluble Surfactant on Turbulent Bubbly Flows in Vertical Channels. This manuscript has already been submitted and we received comments from two reviewers. We have revised the manuscript in response to those comments and recently sent in the revised version. Prof. Muradoglu worked with Tryggvason on numerical methods for soluble surfactants and was part of conceptualizing and getting the present study started during a sabbatical at the University of Notre Dame. He was, however, not funded by CASL.

The other two manuscripts are:

J. Lu and G. Tryggvason. Bubble injection into turbulent upflow in a vertical channel.

J. Lu and G. Tryggvason. DNS of multifluid flows in a vertical channel undergoing topology changes.

Both manuscripts are concerned with turbulent upflow in vertical rectangular channels and the titles describe the content relatively accurately. While we have somewhat self-contained drafts of both manuscripts we are hoping to include some aspects of the data processing described in the last section in both manuscripts and have put them on hold while we explore how to use POD and DMD decompositions to cast light on the structure of the flow. We expect at least one and possibly both to have been submitted by the end of the year.

\section{Conclusions}

As the continued research effort, the present milestone report has focused on the following tasks: (i) the verification of developed advanced analysis method for interface tracking simulations; (ii) The high-fidelity numerical investigation about the impact of spacer grid and mixing vanes on single-phase turbulent flows. Before the further applications of advanced bubble tracking method in more realistic and complicated reactor geometries, the related numerical techniques have been verified. It has been demonstrated that the bubble tracking simulation can provide accurate information regarding the bubble dynamics and flow conditions. Meanwhile, the recent efforts in DNS study of single-phase turbulent flows have been also presented in several PWR subchannel geometries. The mean velocity profile in regular subchannel (case G1) exhibits typical characteristics of turbulent law of the wall. However, the mean velocity profile obtained at the downstream location of case G2 (with SGMV) is clearly different as the velocity magnitude sees a significant drop at the domain centerline due to centrifugal effect produced by the mixing vanes. By assuming the universality of turbulent viscous sublayer, further analysis reveals that the friction velocity in case G2 is $40 \%$ larger than that in case G1. The influence of SGMV would decay as the flow moves to the downstream direction after the SGMV. 
The channel flow data has helped us gain important insight into the dynamics of bubbly upflow, including the dependency of the flow structure on the deformability of the bubbles. These studies have also shown that while surfactants have significant impact on the motion of nearly spherical bubbles, the effect is less for deformable bubbles. Thus, we have most recently focused on bubbles that are sufficiently deformable so that effects of surfactants should be small. The output from one such simulation has been gathered in a database that is being made available to researchers interested in testing out conventional and new turbulence models. The results from the channel flow are also being used to explore the applicability and use of various statistical approaches, some of which have been successfully used for single-phase turbulence.

As we move forward towards simulations with more realistic conditions, DNS results will make much greater contributions in guiding lower-fidelity simulations and engineering designs. The observed quantifiable increase in shear stress in the wake region of the spacer grid is important not only for Reynolds-averaged turbulence modeling approach (as many models are sensitive to shear-stress normalized mesh resolutions), but also to quantify the heat transfer enhancements in operating reactors during single phase and boiling regimes. 


\section{References}

[1] Kolmogorov, A.N., (1941). "The Local Structure of Turbulence in Incompressible Viscous Fluid for Very Large Reynolds Numbers" Dokl. Akad. Nauk SSSR, 30, 299-303.

[2] Turinsky, P.J. and D.B. Kothe, (2016). "Modeling and simulation challenges pursued by the Consortium for Advanced Simulation of Light Water Reactors (CASL)" Journal of Computational Physics, 313, 367-376.

[3] Jansen, K.E., (1999). "A stabilized finite element method for computing turbulence" Comput. Methods Appl. Mech. Eng., 174(3), 299-317.

[4] Rasquin, M., C. Smith, K. Chitale, E.S. Seol, B.A. Matthews, J.L. Martin, O. Sahni, R.M. Loy, M.S. Shephard and K.E. Jansen, (2014). "Scalable Implicit Flow Solver for Realistic Wing Simulations with Flow Control" Computing in Science \& Engineering, 16(6), 13-21.

[5] Fang, J., M. Rasquin and I.A. Bolotnov, (2017). "Interface tracking simulations of bubbly flows in PWR relevant geometries" Nucl. Eng. Des., 312, 205-213.

[6] Lu, J. and G. Tryggvason, (2008). "Effect of bubble deformability in turbulent bubbly upflow in a vertical channel" Physics of Fluids (1994-present), 20(4), 040701.

[7] Fang, J., (2016). "Development of Advanced Analysis Toolkit for Turbulent Bubbly Flow Simulations.".

[8] Fang, J., M.D. Zimmer, I.A. Bolotnov, J. Lu and G. Tryggvason, (2016). "ITM/DNS for high void fraction flows and development of advanced analysis and machine learning algorithms" CASL milestone technical report.

[9] Ninokata, H., N. Atake, E. Baglietto, T. Misawa and T. Kano, (2004). "Direct numerical simulation of turbulent flows in a subchannel of tight lattice fuel pin bundles of nuclear reactors" Annual Report of the Earth Simulator Center April, 2005.

[10] Moser, R.D., J. Kim and N.N. Mansour, (1999). "Direct numerical simulation of turbulent channel flow up to $R e=590 "$ Phys. Fluids, 11(4), 943-945.

[11] Bolotnov, I.A., (2013). "Influence of Bubbles on the Turbulence Anisotropy" ASME J. Fluids Eng., 135(5), 051301.

[12] Lumley, J.L., (1967). "The structure of inhomogeneous turbulent flows" Atmospheric turbulence and radio wave propagation, 790, 166-178.

[13] Sayadi, T. and P.J. Schmid, (2016). "Parallel data-driven decomposition algorithm for large-scale datasets: with application to transitional boundary layers" Theor. Comput. Fluid Dyn., 30(5), 415-428.

[14] Jovanović, M.R., P.J. Schmid and J.W. Nichols, (2014). "Sparsity-promoting dynamic mode decomposition" Phys. Fluids, 26(2), 024103. 